\title{
Characteristics of merging at the magnetopause inferred from dayside 557.7-nm all-sky images: IMF drivers of poleward moving auroral forms
}

\author{
N. C. Maynard ${ }^{1}$, W. J. Burke ${ }^{2}$, Y. Ebihara ${ }^{3}$, D. M. Ober ${ }^{4,}$, , G. R. Wilson ${ }^{4,},{ }^{* *}$, K. D. Siebert ${ }^{4,}{ }^{* * *}$, J. D. Winningham ${ }^{5}$, \\ L. J. Lanzerotti ${ }^{6}$, C. J. Farrugia ${ }^{1}$, M. Ejiri ${ }^{3}$, H. Rème ${ }^{7}$, A. Balogh ${ }^{8}$, and A. Fazakerley ${ }^{9}$ \\ ${ }^{1}$ Space Science Center, University of New Hampshire, Durham, NH, USA \\ ${ }^{2}$ Air Force Research Laboratory, Hanscom Air Force Base, MA, USA \\ ${ }^{3}$ National Institute of Polar Research, Tokyo, Japan \\ ${ }^{4}$ ATK Mission Research, Nashua, NH, USA \\ ${ }^{5}$ Southwest Research Institute, San Antonio, TX, USA \\ ${ }^{6}$ New Jersey Institute of Technology, NJ, USA \\ ${ }^{7}$ CESR/CNRS, Avenue du Colonel Roche, 31028 Toulouse, Cedex 4, France \\ ${ }^{8}$ Imperial College, Exhibition Road, London SW7 2BW, UK \\ ${ }^{9}$ Mullard Space Science Laboratory, Dorking, Surrey RH5 6NT, UK \\ ${ }^{*}$ now at: Boston College, Chestnut Hill, MA, USA \\ ${ }^{* *}$ now at: Center for Space Plasma and Aeronomic Research, University of Alabama in Huntsville, Huntsville, AL, USA \\ ${ }^{* * *}$ now at: SPARTA, Inc., Nashua, NH, USA
}

Received: 5 December 2005 - Revised: 8 September 2006 - Accepted: 20 September 2006 - Published: 22 November 2006

\begin{abstract}
We combine in situ measurements from Cluster with high-resolution $557.7 \mathrm{~nm}$ all-sky images from South Pole to investigate the spatial and temporal evolution of merging on the dayside magnetopause. Variations of $557.7 \mathrm{~nm}$ emissions were observed at a $6 \mathrm{~s}$ cadence at South Pole on 29 April 2003 while significant changes in the Interplanetary Magnetic Field (IMF) clock angle were reaching the magnetopause. Electrons energized at merging sites are the probable sources for $557.7 \mathrm{~nm}$ cusp emissions. At the same time Cluster was crossing the pre-noon cusp in the Northern Hemisphere. The combined observations confirm results of a previous study that merging events can occur at multiple sites simultaneously and vary asynchronously on time scales of $10 \mathrm{~s}$ to $3 \mathrm{~min}$ (Maynard et al., 2004). The intensity of the emissions and the merging rate appear to vary with changes in the IMF clock angle, IMF $B_{X}$ and the dynamic pressure of the solar wind. Most poleward moving auroral forms (PMAFs) reflect responses to changes in interplanetary medium rather than to local processes. The changes in magnetopause position required by increases in dynamic pressure are mediated by merging and result in the generation of PMAFs. Small (15-20\%) variations in dynamic pressure of the solar wind are sufficient to launch PMAFs. Changes in IMF $B_{X}$ create magnetic flux compressions and rarefactions in the solar wind. Increases (decreases) in IMF $B_{X}$
\end{abstract}

Correspondence to: N. C. Maynard

(nelson.maynard@unh.edu) strengthens $|\mathbf{B}|$ near northern (southern) hemisphere merging sites thereby enhancing merging rates and triggering PMAFs. When correlating responses in the two hemispheres, the presence of significant IMF $B_{X}$ also requires that different lagtimes be applied to ACE measurements acquired $\sim 0.1 \mathrm{AU}$ upstream of Earth. Cluster observations set lag times for merging at Northern Hemisphere sites; post-noon optical emissions set times of Southern Hemisphere merging. Allsky images and magnetohydrodynamic simulations indicate that merging occurs in multiple discrete locations, rather than continuously, across the dayside for southward IMF conditions in the presence of dipole tilt. Matching optical signatures with clock-angle, $B_{X}$, and dynamic pressure variations provides new insights about interplanetary control of dayside merging and associated auroral dynamics.

Keywords. Magnetospheric physics (Auroral phenomena; Magnetosphere-ionosphere interactions; Solar windmagnetosphere interactions)

\section{Introduction}

Magnetic merging at the dayside magnetopause is the dominant mechanism responsible for coupling solar-wind energy and momentum to near Earth space along newly opened field lines that connect to the ionosphere via the cusps (Dungey, 1961). Numerous satellites have observed merging

Published by Copernicus GmbH on behalf of the European Geosciences Union. 
signatures at a variety of magnetopause locations (e.g., Paschmann et al., 1979; Sonnerup et al., 1981, 1990; Gosling et al., 1991; Phan et al., 1996, 2001; Scudder et al., 2002; Mozer et al., 2003). Such observations only apply to local conditions but cannot support synoptic assessments about either their spatial-temporal extents or the variability of active merging.

Since the magnetopause maps to the ionosphere through the cusps, phenomena occurring at the equatorward boundaries of the cusps provide two-dimensional images of merging activity. With the cusp in darkness above Ny-Ålesund in the Svalbard archipelago, Maynard et al. (2001b) observed $557.7 \mathrm{~nm}$ emissions that intensified and dimmed in response to variations in the interplanetary magnetic field (IMF). Since the lifetime of the ${ }^{1} \mathrm{~S} \rightarrow{ }^{1} \mathrm{D}(557.7 \mathrm{~nm})$ transition is $1 \mathrm{~s}$, Maynard (2003) suggested that all-sky images of the cusp provide "television pictures" of large-scale merging at the magnetopause. Subsequently Maynard et al. (2004) showed that $557.7 \mathrm{~nm}$ cusp emissions above Ny-Ålesund are localized rayed structures that appear and vanish at particular locations on time scales ranging from $30 \mathrm{~s}$ to several minutes. Structures may occur simultaneously, but asynchronously, at multiple locations, then change positions in local time in response to changes in the interplanetary magnetic field (IMF) clock angle $\theta$. Particle measurements acquired during a fortuitous DMSP overflight of an auroral structure above Svalbard showed that electrons with energies between 0.5 and a few $\mathrm{keV}$ were the sources of $557.7 \mathrm{~nm}$ emissions. Maynard et al. (2005) used Polar data to show that electrons with similar energies constitute the high-energy tails of the suprathermal electron distributions found on field lines connected to active merging sites.

Between 15:00 and 17:00 UT on 29 April 2003, all sky imagers at the South Pole Station $\left(-74.14^{\circ}\right.$ magnetic latitude, midnight MLT=03:34 UT) monitored the dynamics of several poleward moving auroral forms (PMAFs) (Sandholt et al., 1986) that followed changes in $\theta$, IMF $B_{X}$ and $P_{S W}$. Simultaneously, the four-satellite configuration of Cluster crossed the magnetopause into the magnetosheath, poleward of the northern hemisphere cusp. Plasma and IMF signatures observed by the Advanced Composition Explorer (ACE) near $L_{1}$ and by Cluster in the magnetosheath establish lag times appropriate for the northern hemisphere.

As demonstrated below, most $557.7 \mathrm{~nm}$ and $630.0 \mathrm{~nm}$ PMAF activity observed at South Pole Station are related to changes in $\theta$, IMF $B_{X}$ and $P_{S W}$, either individually or in combination. Interpreting super-thermal electron tails as surgical indicators of active merging, we link $557.7 \mathrm{~nm}$ allsky observations and Cluster measurements, to show that: (1) changes in the magnetopause configuration following increases in $P_{S W}$ are largely accomplished though magnetic merging, (2) IMF $B_{X}$ variations increase (decrease) the magnetic flux presented to merging sites and thereby affect merging rates, (3) IMF clock-angle fluctuations alter the locations of merging sites in the northern and southern hemisphere and impact them at different times, and (4) magnetic merging is locally discrete and time dependent but occurs at multiple sites simultaneously. Since this involves many diverse topics, the remainder of the introduction provides necessary background information relevant to each.

To establish causal connections between magnetic merging at the magnetopause and $557.7 \mathrm{~nm}$ emissions in the ionospheric projections of the cusps, it is necessary to demonstrate that merging sites produce adequate fluxes of energetic electron with access to the appropriate locations. Gosling et al. (1990) suggested a two-dimensional picture of mergingsite geometries with a separator (merging) line between two freshly opened field lines that extend to the ionosphere below and to the solar wind above, respectively called inner and outer separatrices. Near separator lines electron gyrotropy breaks allowing particles initially trapped in the outer plasma sheet to lose connection with closed field lines and then reattach to newly opened flux at random pitch angles. Their high velocities $(20000 \mathrm{~km} / \mathrm{s})$ carry electrons in the suprathermal "tails" of distribution functions rapidly away from the separators. Thus, energetic electrons maintain closer proximity to separatrices than slower moving ions. Sonnerup et al. (1981) and Scudder et al. (1984) reported magnetically aligned low$\mathrm{keV}$ electrons, observed near the magnetopause current layer, as heat fluxes. Near the magnetopause the Polar satellite often detects similar electron fluxes at large distances from active separator sources (Maynard et al., 2005). Because of their high velocities, electron tails are observable immediately after they emerge from separator regions. Unless replenished by continued merging, newly opened flux tubes would quickly empty of electron-tail populations. Thus, electron tails act as surgical indicators of active merging.

Electrons exiting merging regions along inner separatracies intersect the ionosphere very close to the open-closed field-line boundary, where they excite structured $557.7 \mathrm{~nm}$ auroral emissions in the ionosphere (Maynard et al., 2004). Because their high speeds allow quick reflection from the ionosphere, tail electrons within the magnetosphere move in both directions along field lines (Gosling et al., 1990; Maynard et al., 2005). When the IMF has a significant $B_{Y}$ component, the separatrix geometry becomes more complex than the simple picture presented by Gosling et al. (1990). In such cases magnetic mapping between merging sites and the conjugate northern and southern cusps remains intelligible, albeit more complicated. However, the temporal and spatial properties of energetic electron tails are similar (Maynard et al., 2005).

Cowley and Lockwood (1992) suggested that merging is intermittent, occurring in bursts, separated by quiescent gaps at particular locations. Observed repetition rates of $\sim 8 \mathrm{~min}$ are similar to those of flux transfer events (FTEs) near the subsolar magnetopause (Russell and Elphic, 1979). The Cowley-Lockwood merging bursts appear to be separated by longer temporal gaps and are wider in azimuth than the $\approx 1 R_{E}$ sizes estimated for typical FTEs. 
Maynard et al. (2004) estimated that merging activations last between $\approx 30 \mathrm{~s}$ and several minutes from the durations of $557.7 \mathrm{~nm}$ cusp emissions. However, their spatial scales were smaller than those postulated by Cowley and Lockwood, and many were detected simultaneously but asynchronously. Polar observations of field-aligned electron tails also suggest that merging activations occur on time scales lasting from a few tens of seconds to several minutes (Maynard et al., 2005). During magnetopause crossings by Interball-Tail, Vaisberg et al. (2001) found that the low-latitude boundary layer became highly structured while the IMF $B_{Z}$ was southward. Transients, characterized by bi-directional keV electron fluxes lasting $30 \mathrm{~s}$ to $5 \mathrm{~min}$, led Vaisberg et al. (2001) to interpret them as occurring on closed field lines. Others (e.g. Lockwood, 1997; Maynard et al., 2001a) concluded that in such cases the low-latitude boundary layer is mostly a region of open field lines.

Ionospheric signatures of FTEs have been studied for the past two decades. Of interest here is an association of FTEs with poleward moving auroral forms (PMAFs) first described by Sandholt et al. (1986). PMAFS are structures found in the keogram displays of meridian scanning photometer data acquired near magnetic noon. They first appear as intensifications of $630.0 \mathrm{~nm}$ emissions near the cusps equatorward boundary then move poleward with time. Detecting PMAFs requires a significant IMF $B_{Y}$; they vanish as the IMF clock angle $\theta$ approaches $180^{\circ}$ (Sandholt and Farrugia, 2002). $557.7 \mathrm{~nm}$ intensifications occur at the roots of PMAF structures along the equatorward edge of the cusp. FTEs move toward dawn (dusk) during periods of positive (negative) IMF $B_{Y}$ before being swept back poleward and over the mantle. Sandholt et al. $(2002,2004)$ postulated that $557.7 \mathrm{~nm}$ intensifications at the cusps equatorward boundary reflect electron precipitation from the low-latitude boundary layer and/or the boundary plasma sheet (BPS) (Newell and Meng, 1994).

Increased dynamic pressure in the solar wind $\left(P_{S W}\right)$ also creates transient Chapman-Ferraro currents at the magnetopause, the surface where $\mathbf{J} \times \mathbf{B}$ forces balance those exerted by pressure gradients. Large transient changes in $P_{S W}$ distort the magnetopause and launch Earthward moving fast-mode waves and Alfvén waves that carrying field-aligned currents (see Southwood and Kivelson, 1990; Kivelson and Southwood, 1991; Sibeck, 1990; Sibeck et al., 2003). Consequent fast-mode compression waves have been observed at geosynchronous orbit (Korotova et al., 1999), as well as in the high and low latitude ionosphere. Pressure pulse models generally involve variations that are the same order of magnitude as the background pressure. In the SouthwoodKivelson model longitudinally dependent pressure changes at the magnetopause generate pairs of field-aligned currents that couple to the incompressible ionosphere. Magnetic impulse events (MIEs)(e.g. Lanzerotti et al., 1986; Konik et al., 1994; Kataoka et al., 2003) and traveling convection vortices (TCVs) (Friis-Christensen et al., 1988), observed in the high-latitude ground magnetograms, couple to pressure pulses through pairs of field-aligned currents (Zesta et al., 1999; Sibeck et al., 2003). TCVs are much larger in scale than FTEs. They move away from initial points of contact as expected for surface variations propagating away from highpressure sources. MIEs may also be produced from sources other than pressure variations, and they have been observed at times when there are small IMF cone angles (Kataoka et al., 2001).

The pressure-pulse model suggested by Sibeck (1990) predicts that spiral/orthospiral orientations of the IMF control the local times at which events occur. For spiral IMF orientations $\left(+B_{X}\right.$ and $-B_{Y}$ or $-B_{X}$ and $\left.+B_{Y}\right)$, dynamic pressure variations convect to the bow shock where they launch pressure variations that propagate through the magnetosheath and strike extended regions of the postnoon magnetopause before sweeping across the prenoon magnetopause (Sibeck et al., 2003). Initially plasma motion in the low-latitude boundary layer may be sunward, before being carried down the dawn flank. In the ionosphere this translates to azimuthal motion. Most spiral-IMF driven events observed in the prenoon sector move dawnward (Korotova et al., 2004). However, Sibeck et al. (2003) showed that for a spiral IMF the postnoon first impact created activity that propagated past noon toward dawn. Sanny et al. (1996) studied dayside magnetospheric responses to observed increases in the IMF strength and bi-polar fluctuations in $B_{X}$ exceeding $4 \mathrm{nT}$. They argue that pressure pulses are the dominant cause of events observed in the closed field line part of the outer magnetosphere. Konik et al. (1994) identified $>40 \mathrm{nT}$ impulse events in ground magnetograms that they interpreted as primarily signatures of sporadic reconnection. Dynamic pressure may have played a role in a small fraction of the cases. Since the reported events occurred at high magnetic latitudes, most likely they were detected poleward of the open-closed boundary.

Maynard et al. (2000, 2001b) demonstrated the importance of $B_{X}$ for estimating lag intervals for interplanetary electric field (IEF) effects to propagate from the first Lagrangian point $\left(L_{1} ; \approx 0.01 \mathrm{AU}\right.$ upstream from the Earth) to the dayside magnetopause. They reported lag times shorter than the advection times for correlated behavior between variations in the IMF and electric-field responses observed in the northern ionosphere by sounding rockets launched from Svalbard. External circumstances indicated that merging near the southern-hemisphere cusp was responsible for the prenoon activity in the northern ionosphere. A longer lag was required to explain postnoon activity driven by merging near the northern hemisphere cusp. The tilts of IEF planes of constant phase are controlled by the strength of $B_{X}$. Weimer et al. (2002) validated the concept using IMF measurements from four satellites in the solar wind and demonstrated that the tilts of IEF phase planes can change significantly in tens of $\min$. 


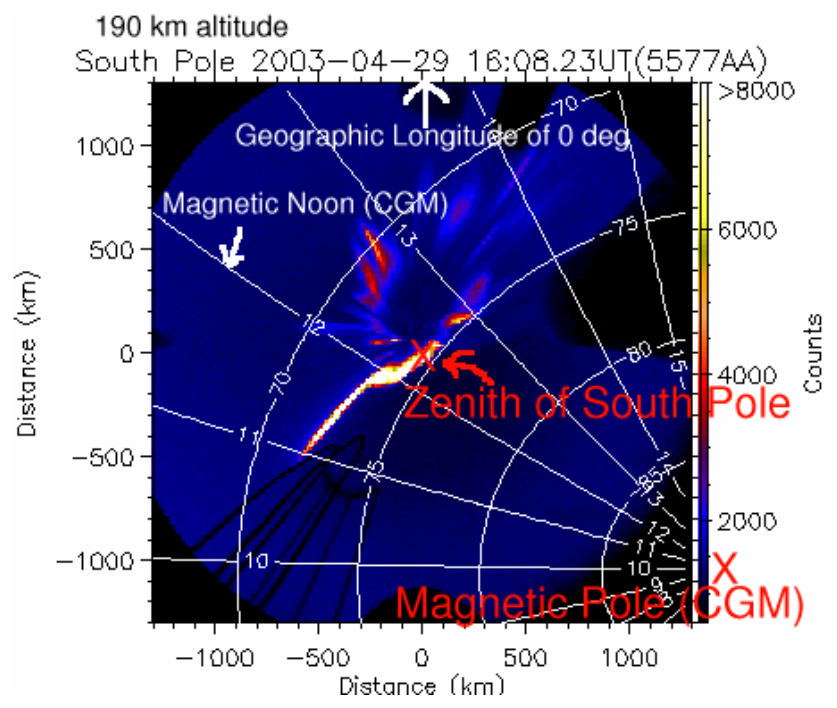

Fig. 1. Sample $557.7 \mathrm{~nm}$ all-sky image mapped onto a spherical shell at $190 \mathrm{~km}$ altitude above the South Pole. The perspective is that of an observer looking down from above. The red Xs at the center of the image and to its right mark the locations geographic zenith and the magnetic pole, respectively. The direction of the Greenwich prime meridian $\left(0^{\circ}\right.$ geographic longitude) is indicated at the top of the image. "Distance" labels along the ordinate and abscissa signify arc lengths from the point that intersects South Pole Stations zenith at $190 \mathrm{~km}$ altitude. White lines indicate magnetic latitude and MLT in the CGM coordinates. In-figure labels " -70 " and " 12 " reference CGM latitude $-70^{\circ}$, and MLT noon. Because emissions at particular locations on the sphere have vertical extents, they may appear as rays that point toward magnetic zenith. The black structure in the lower left and the occlusions in the upper right detail regions of interference in the all-sky field of view. The background appears relatively bright compared with most other presented images. This is an instrumental effect due the very strong central emissions.

Maynard et al. (2003) used Polar and Cluster data to show that merging typically occurs at high latitudes for $0<|\theta|<150^{\circ}$. As a result, IMF $B_{Y}$ bifurcates the cusp according to source regions (Maynard et al, 2001b; Coleman et al, 2001; Roger et al., 2003). Merging locations follow the prescription of Crooker (1979) where the magnetic field lines are nearly anti-parallel. For IMF $B_{Y}$ positive (negative) highlatitude merging sites are near the postnoon (prenoon) rim of the rim of northern-hemisphere cusp. In the southern hemisphere the opposite relationship applies. In the $B_{Y}$ negative cases examined in this paper, optical emissions observed at postnoon (prenoon) local times reflect merging events near the corresponding rim of the cusp in the southern (northern) hemisphere. When merging at high latitudes dominates, the phase-plane tilts described above, yield significantly different lag times for merging signatures in the northern and southern hemispheres, under the influence of IMF $B_{X}$. Conversely, models demanding that only one component of $\mathbf{B}$ needs to be anti-parallel (Sonnerup, 1974), predict that merg- ing extends along tilted lines that pass through subsolar region (Gonzales and Mozer, 1974; Moore, 2002) and do not require significant timing differences between the two hemispheres.

\section{Measurement and modeling techniques}

\subsection{All-sky Images from South Pole Station}

On 23 April, and from 25 April to 2 May 2003, hightemporal resolution $557.7 \mathrm{~nm}$ all-sky images were acquired at South Pole Station during Polar and Cluster overflights. This paper focuses on the images taken on 29 April 2003. A full sequence for image acquisition takes $42 \mathrm{~s}$. Four consecutive $557.7 \mathrm{~nm}$ images with exposure times of $4 \mathrm{~s}$ are presented at $6 \mathrm{~s}$ separations. The remaining $20 \mathrm{~s}$ of a cycle are spent taking images at $630.0 \mathrm{~nm}$ by rotating a filter wheel and focusing the lens. During this interval measurements were only made at wavelengths of $557.7 \mathrm{~nm}$ and $630.0 \mathrm{~nm}$. We present data sequences of four $557.7 \mathrm{~nm}$ images $6 \mathrm{~s}$ apart, followed by $20 \mathrm{~s}$ gaps before the next 4 -image sequence.

Figure 1 shows an illustrative $557.7 \mathrm{~nm}$ image mapped onto a geocentric spherical shell at $190 \mathrm{~km}$ altitude, and transposed to the perspective of an observer looking down from above the South Pole Station. The expected sources of the emissions, electron tails from active merging, have energies between about $500 \mathrm{eV}$ and several $\mathrm{keV}$ (Maynard et al., 2005). These electrons lose their energies through the ionization and excitation of neutrals at altitudes between 200 and $125 \mathrm{~km}$, producing $557.7 \mathrm{~nm}$ emissions (Rees, 1963). We chose $190 \mathrm{~km}$ near the upper end of this range because the number flux is greater at lower energies. We also considered projections to $150 \mathrm{~km}$. While the affected areas are contracted when compared with those shown, our conclusions are unaltered. The red Xs at the center of the image and to its right mark the locations of the geographic zenith and the magnetic pole, respectively. The direction of the prime meridian $\left(0^{\circ}\right.$ geographic longitude) is indicated at the top of the image. "Distance" labels along the ordinate and abscissa signify arc lengths from the point that intersects the zenith at South Pole Station at $190 \mathrm{~km}$ altitude. White lines indicate magnetic latitude and MLT in CGM coordinates. In-figure labels " -70 " and "12" reference CGM latitude $-70^{\circ}$ and MLT noon, respectively. Because emissions at particular locations on the sphere have vertical extents, they may appear as rays that point toward magnetic zenith. Points located on the image farthest from the magnetic zenith give the locations where auroral field lines intersect the mapping sphere at $190 \mathrm{~km}$ altitude. The long bright structure between 11:00 and 12:00 MLT may be continuous or have multiple components that align toward magnetic zenith. We also present, below, meridian scans (or keograms) excerpted from all-sky images at 557.7 and $630.0 \mathrm{~nm}$ along the magnetic meridian 
passing through the South Pole Station zenith and the south magnetic pole.

\subsection{Interplanetary plasmas and fields}

The Advanced Composition Explorer (ACE) spacecraft monitors interplanetary conditions while flying in a halo orbit around $L_{1}$ in front of the Earth. The solar wind velocity was measured by the Solar Wind Electron, Proton, and Alpha Monitor (SWEPAM) (McComas et al., 1998). A tri-axial fluxgate magnetometer measured the vector interplanetary magnetic field (Smith et al., 1998). We also use data from the Magnetic Field Investigation (Lepping et al., 1995) and the Solar Wind Experiment (Ogilvie et al., 1995) on the Wind spacecraft as crosschecks. Wind was executing a distant prograde orbit. While it generally had large $Y$ coordinates, in our interval it had similar $Y$ coordinates as ACE. The GSE locations of ACE and Wind for the event presented here are $(233,40,-21) R_{E}$ and $(254,37,18) R_{E}$, respectively.

\subsection{Cluster}

The four Cluster spacecraft, with Cluster 4 in the lead and Cluster 3 trailing, passed through the northern hemisphere cusp while the optical measurements were being made at South Pole. The nominal separation distances between the different spacecraft was $\sim 5000 \mathrm{~km}$. Magnetic field measurements were made on each of the Cluster spacecraft by triaxial fluxgate magnetometers (Balogh et al., 2001). The electric field and wave instrument (EFW) monitored both electric field components in the ecliptic plane using biased double probes (Gustafsson et al., 1997). The Cluster Ion Spectrometer (CIS) experiment provided 3-D ion distributions with mass per unit charge composition using the Composition and Distribution Function (CODIF) analyzer (Rème et al., 2001). Ion measurements are available only from Spacecraft 1, 3, and 4. The Plasma Electron And Current Experiment (PEACE) (Johnstone et al., 1997) uses top-hat electrostatic analyzers to provide high angular resolution measurements of electrons with energies between $0.59 \mathrm{eV}$ and $26.4 \mathrm{keV}$.

\subsection{The Integrated Space Weather Prediction Model (ISM)}

The ISM uses standard magnetohydrodynamic (MHD) equations augmented with hydrodynamic equations for a collisionally coupled neutral thermosphere (White et al., 2001). The code transitions seamlessly from pure MHD for plasma in the solar wind and magnetosphere to proper ionosphere/thermosphere equations at low-altitudes. ISM equations are solved within a three-dimensional computational domain extending from an interior spherical boundary at the approximate bottom of the E-layer $(100 \mathrm{~km})$ extending up through the magnetosphere and out into the solar wind. The spatial resolution of ISMs computational grid varies from a few hundred kilometers in the ionosphere to several $R_{E}$ near the outer boundary of the computation domain. Grid resolution in the region of the dayside magnetopause is uniform at $0.3 R_{E}$ resolution.

Explicit viscosity in the plasma momentum equation was set to zero. In the Ohms Law equation an explicit resistivity term is added when the current density normal to $\mathbf{B}$ exceeds a specified threshold. In practice, this choice leads to non-zero explicit resistivity near the subsolar magnetopause, and in the nightside plasma sheet. Where needed to maintain numerical stability, dissipation is based on the partial donorcell method (PDM) as formulated by Hain (1987). Dissipation is necessary in the code to approximate non-linear magnetic reconnection. It is important to note that the code solves for the configuration imposed by the boundary conditions by adding dissipation. In general for the simulation presented here, added dissipation on the dayside was accomplished in response to the imposed driving conditions through PDM and not the current dependent resistivity term. Further details describing the ISM code can be found in White et al. (2001). The version of ISM used here is similar except for some differences in the geometry of the computational grid.

\section{April 2003}

\subsection{Interplanetary plasmas and fields}

Between 14:00 and 16:00 UT on 29 April 2003 the solar wind velocity and dynamic pressure measured by ACE were moderate, with small density changes modulating $P_{S W}$ (Fig. 2). The solar wind data in Fig. 2 have been lagged by $55.5 \mathrm{~min}$. The lag was chosen so that the Y-Z clock angles observed by ACE and Cluster 4 match during times when Cluster was in the magnetosheath. Magnetic field measurements are presented in GSM coordinates. For comparison with post-shock magnetic fields, ACE IMF measurements were multiplied by 4.5 . The magnetic field was steady in magnitude $\sim 10 \mathrm{nT}$ (black trace, panel d), but had significant changes in its three components (black traces, panels a, b, c). The IMF clock angle in the GSM Y-Z plane rotated from about $-145^{\circ}$ to $180^{\circ}$. For clock angles near $-145^{\circ}$ we anticipate high-latitude merging to proceed along the pre-noon (post-noon) rim of the northern (southern) hemisphere cusp. IMF $B_{X}$ was positive and comparable in magnitude to $B_{Y}$ and $B_{Z}$.

The Cluster constellation was located in and near the northern hemisphere cusp, on the prenoon side at approximate GSE coordinates of $\left(7.0,-5.2,6.9 R_{E}\right)$. The red traces in panels (a-d) of Fig. 2 give magnetic field measurements at the locations of Cluster 4 . The opposite $B_{X}$ polarities measured by the two spacecraft (panel a) are artifacts of magnetic flux tube draping over the magnetopause.

Cluster 4 crossed the magnetopause current layer between 15:13 and 15:33 UT (see Fig. 2, panel d). Magnetic field and particle-flux measurements indicate that Cluster 4 was first 


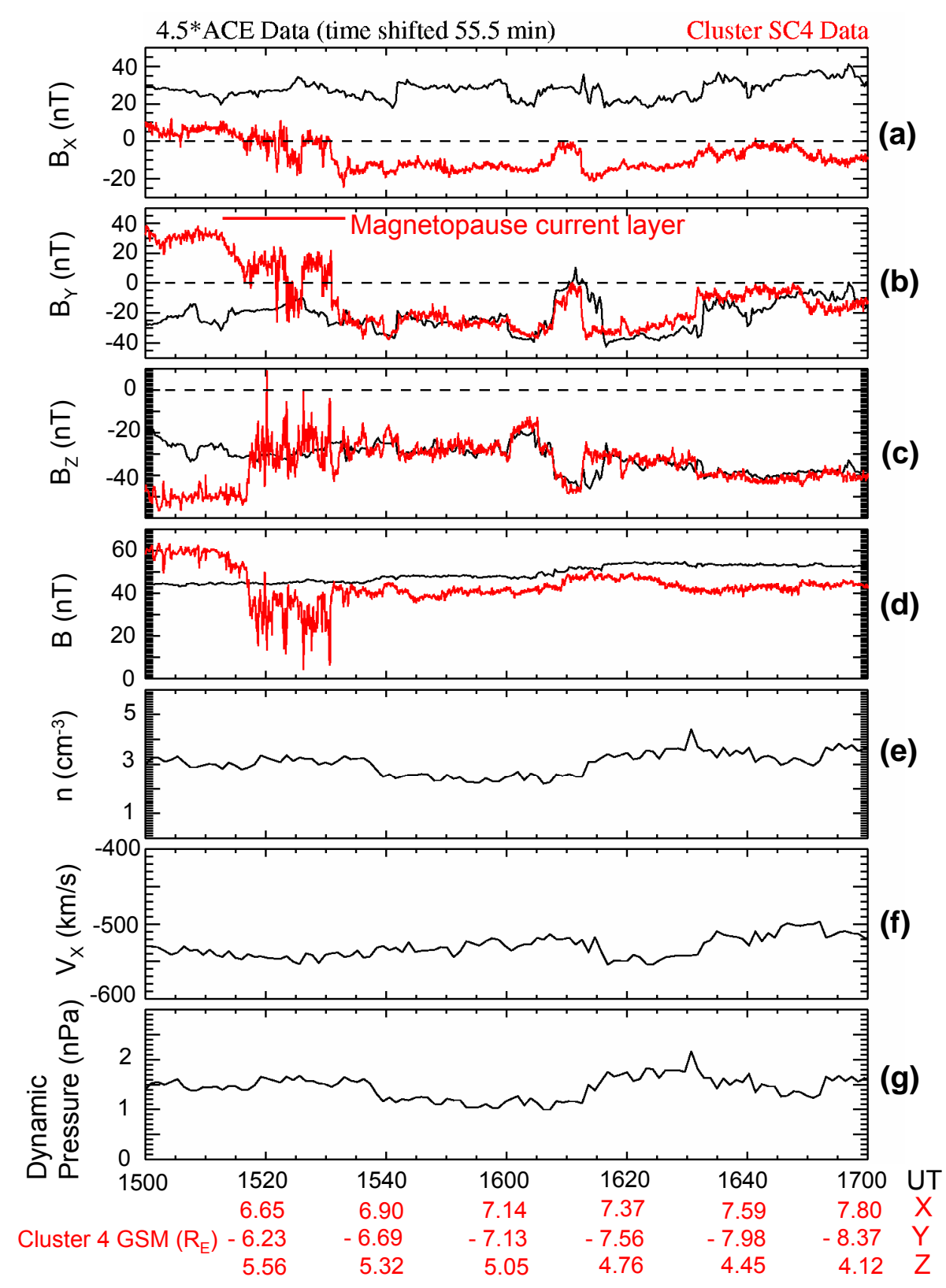

Fig. 2. Interplanetary measurements in GSM coordinates from ACE (black) compared with those from Cluster (red). The ACE magnetic field measurements were multiplied by 4.5 to allow for the jump in magnitude across the bow shock. They were also time shifted by 55.5 min to provide the best overall agreement between the $Y$ and $Z$ components during the interval. While the lag time varies some during the two-hour interval, $55.5 \mathrm{~min}$ gives the best overall lag estimate. In addition to magnitude and three components of $\mathbf{B}$, we also show the solar wind density, velocity, and dynamic pressure as measured at ACE.

to cross the magnetopause, followed by Clusters 1,2 , and 3 (data not shown). Their entrance into the magnetosheath from the magnetotail lobe is most notable in $B_{Y}$ variations. Lesser changes in $B_{X}$ were in phase with $B_{Y}$ variations. These changes in the magnetic field place all four spacecraft poleward of the cusp on the prenoon side. The corresponding Chapman-Ferraro currents had components in the $+\mathrm{X},-\mathrm{Y}$ and $-\mathrm{Z}$ directions.
The significantly positive IMF $B_{X}$, suggests that phase planes in the solar wind that contains $\mathbf{B}$ and the interplanetary electric field are tilted (Maynard et al., 2001; Weimer et al., 2002). Variations in $B_{X}$ alter phase plane tilt angles. Figure 3 is a 2-D cartoon that illustrates the effects of tilt changes in the X-Z plane due to $B_{X}$ modifications. Magnetic field lines represent a time sequence passing ACE, which marches toward the Earth (to the left) with the solar wind motion, 


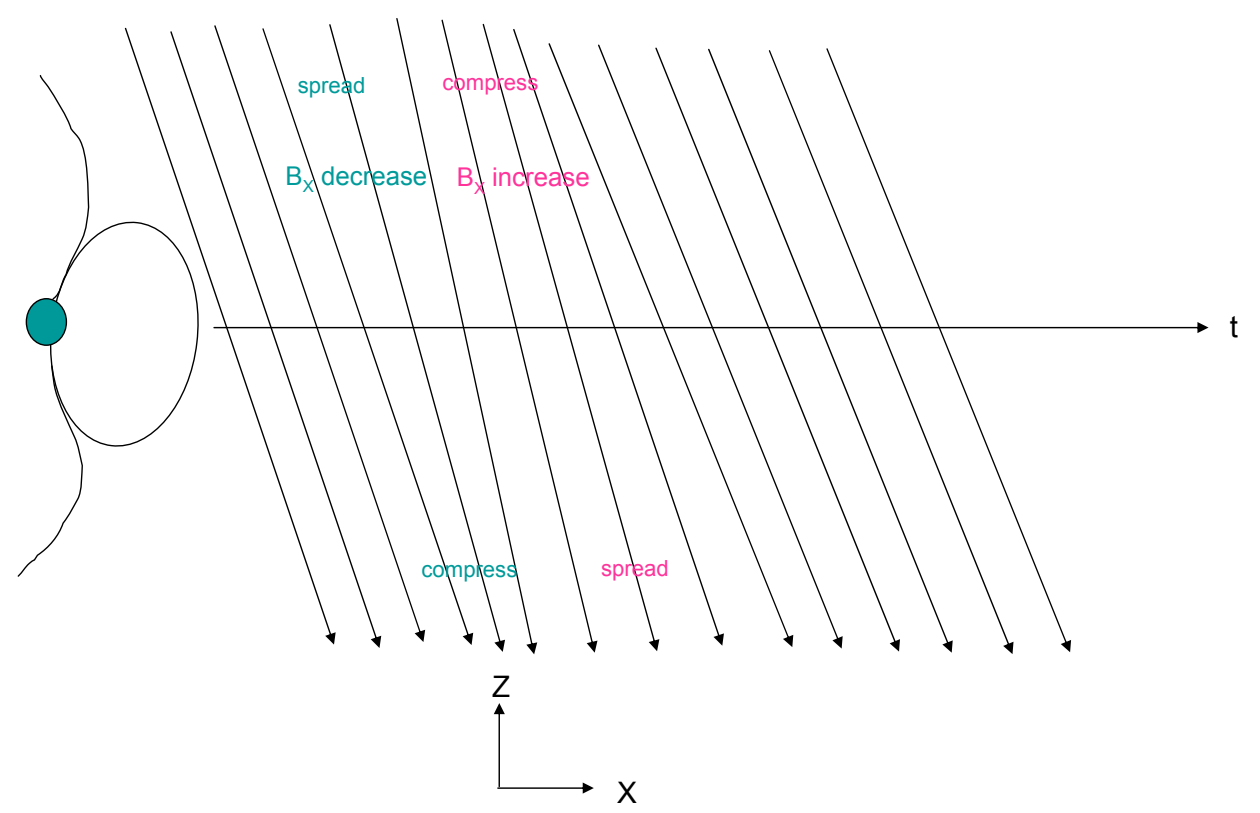

Fig. 3. Cartoon representation of magnetic field compression/spreading as $B_{X}$ changes the tilt of planes of constant IEF phase. Time is to the right along the axis, so that the earlier times reach the magnetosphere first as they flow with the solar wind in the $X$ direction. The bow shock and draping of the magnetic field over the magnetopause, as well as variations in the $Y$ direction, have been omitted for simplicity. Above and below the axis regions of compression (spreading) increase (decrease) the flux presented for merging at a particular location on the magnetopause.

Table 1. ISM simulation input conditions.

\begin{tabular}{lccccccccc}
\hline & & \multicolumn{3}{c}{ IMF } & & Solar & Wind & Dipole & Tilt \\
& $\mathrm{B}_{X}$ & $\mathrm{~B}_{Y}$ & $\mathrm{~B}_{Z}$ & $|\mathrm{~B}|$ & Clock & $\mathrm{V}_{X}$ & $\mathrm{n}$ & $\theta$ & $\phi$ \\
\hline Initial GSE & 6.0 & -6.8 & -5.3 & 10.5 & & -530 & 3 & 29.3 & 31.8 \\
Initial GSM & 6.0 & -5.0 & -7.0 & 10.5 & -145 & & & 24.6 & \\
Final GSE & 6.0 & -2.4 & -8.2 & 10.5 & & -530 & 3 & 29.3 & 31.8 \\
Final GSM & 6.0 & -0.0 & -8.6 & 10.5 & -180 & & & 24.6 & \\
\hline
\end{tabular}

principally in the $-X$ direction. Along the axis labeled $\mathrm{t}$ the lines are equi-spaced, since at ACE all three components varied in a way that only slightly changed (decreased) $|B|$. Above and below the axis magnetic field lines are pushed together or spread apart by the rotations imposed by changes in $B_{X}$ along the axis in this planar approximation. Weimer et al. (2002) demonstrated that the planer approximation holds over scales of $10 \mathrm{~s}$ of $R_{E}$, even with time variations on scales of minutes. The cartoon illustrates two points. First, because of the phase plane tilt with positive $B_{X}$ and negative $B_{Z}$, northern-hemisphere merging sites are reached before those in the southern hemisphere. Second, an increase (decrease) in $B_{X}$ compresses (spreads) magnetic flux to the north and spreads (compresses) it to the south of the hinge point.

\subsection{Magnetopause configuration}

Previously reported ISM simulations indicate that dipole tilt causes merging sites to move away from the cusp closest to the Sun (Maynard et al., 2003). At 16:00 UT the northern dipole axis tilts toward the Sun in its daily rotation. During this late April interval, a month after equinox, the dipole axis has an added, seasonal sunward tilt. The total tilt angle from the GSE $\mathrm{Z}$ axis was $24.5^{\circ}$ during the time of interest. In this case merging sites tend to move toward the southern hemisphere. Pre-noon merging sites near the northernhemisphere cusp move toward the equator, and equatorial merging sites move to higher magnetic latitudes in the southern hemisphere. The strongly positive IMF $B_{X}$ and sunward dipole tilt places first IMF contact with the magnetosphere near the northern-hemisphere cusp. IMF field lines then drape around the magnetosphere to lower latitudes and to southern hemisphere merging sites. 


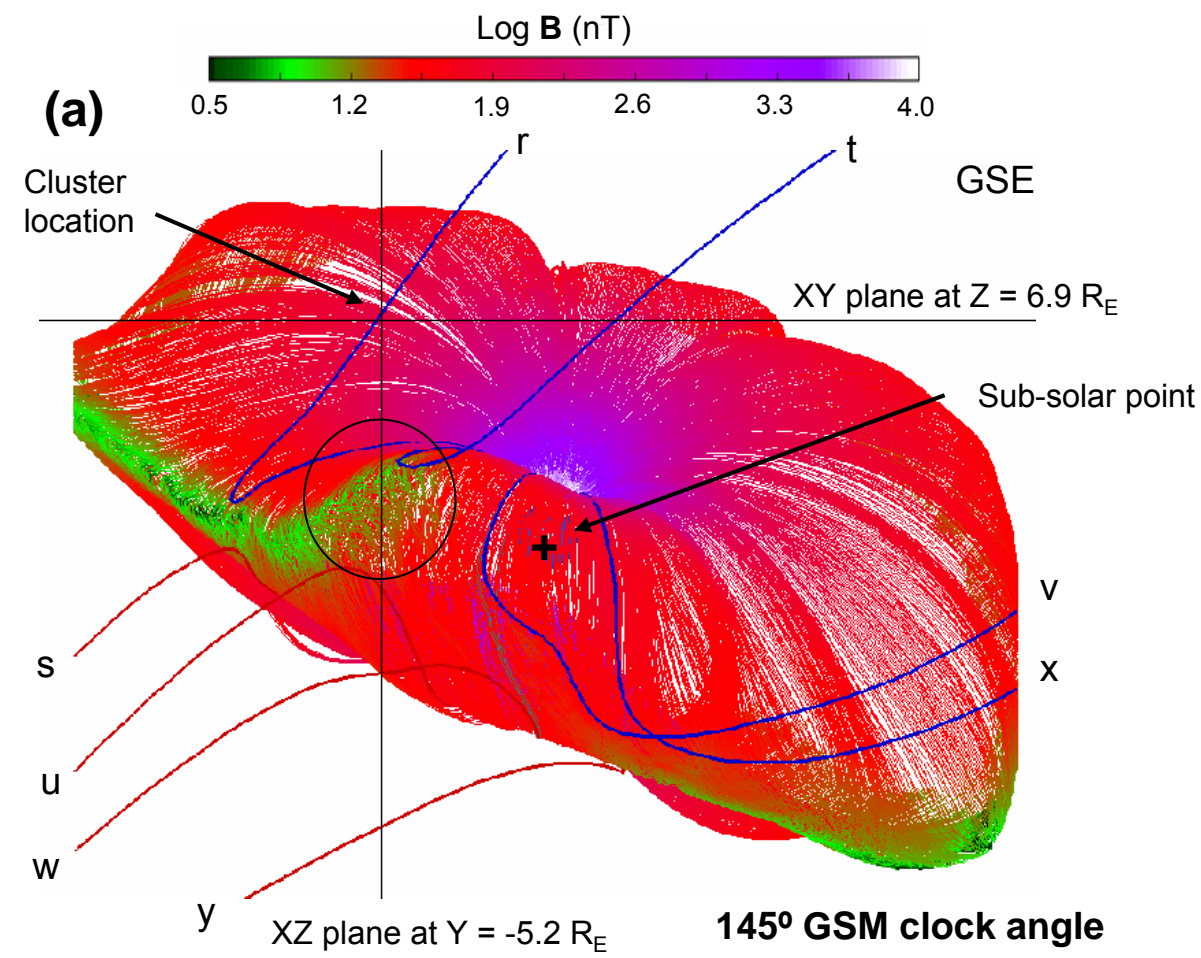

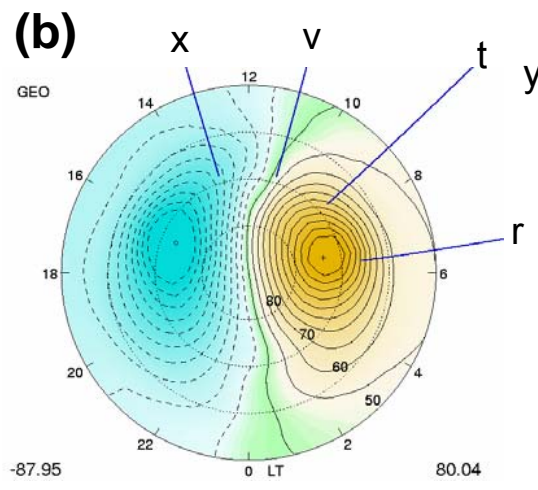

Northern Hemisphere

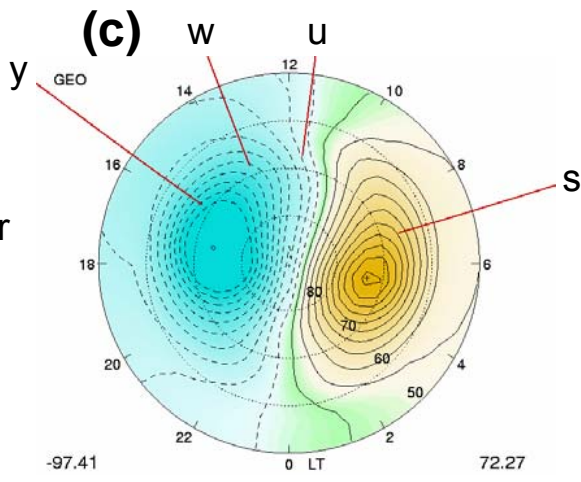

Southern Hemisphere

Potential (kV)

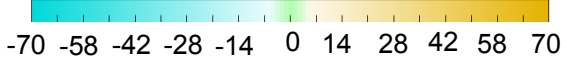

Fig. 4. ISM simulation results with input conditions similar to the average conditions observed during the optical-measurement period. (a) A 3-dimensional view of the magnetopause in GSE coordinates viewed from the Sun. The magnetopause is defined by the sets of last closed field lines mapped from the northern and southern hemisphere ionospheres. Field lines are colored with the log of the magnitude of $|\mathbf{B}|$. Regions of low field strength (green and black) on the magnetopause surface are proxies for probable merging sites. The location of Cluster in this 3-dimensional view is at the intersection of the XY plane (line) at $Z=6.9 R_{E}$ and the $X Z$ plane (line) at $Y=-5.2 R_{E}$. Four pairs of first open field lines, labeled $\mathrm{r}$ through $\mathrm{y}$, highlight regions of importance in interpreting the $557.7 \mathrm{~nm}$ emissions (see text). The foot-points of the field lines in the northern and southern hemisphere ionospheres are shown in (b) and (c) with the respective potential patterns as background.

An ISM simulation of the conditions was conducted using the input parameters listed in Table 1. The simulation ran for $2 \mathrm{~h}$ and $10 \mathrm{~min}$ at a GSM clock angle of $145^{\circ}$. The clock angle then changed to $180^{\circ}$, and the new configuration was allowed to propagate past the magnetopause. Results of the initial-state simulation are given in Fig. 4. Results applicable after the clock-angle rotation to $180^{\circ}$ are discussed in conjunction with Fig. 10.

Figure 4a represents the magnetopause (viewed from the Sun) as a surface of last closed field lines, colored by the 


\section{South Pole: April 29, 2003}
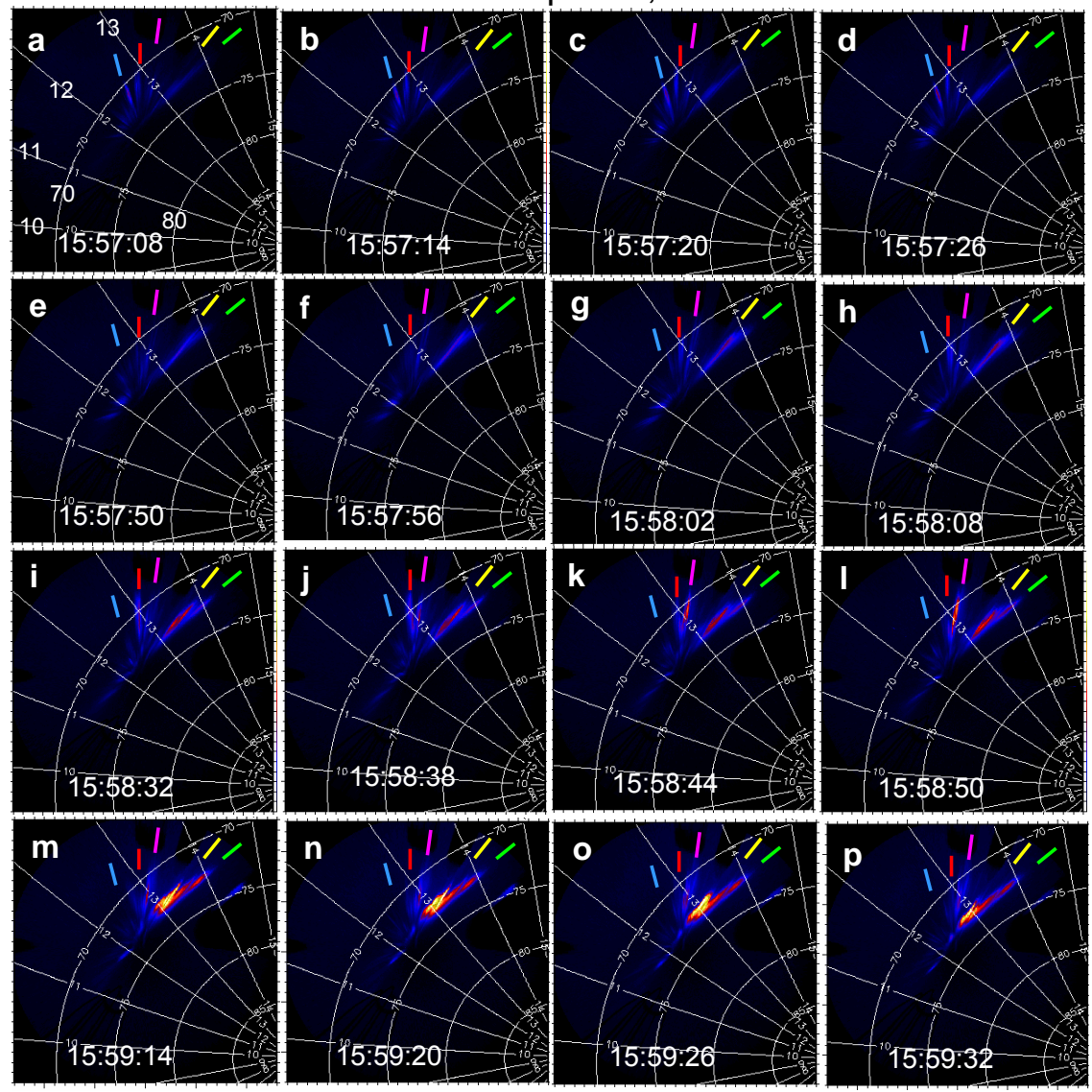

Fig. 5. South Pole Station all-sky images at $557.7 \mathrm{~nm}$ in the format of Fig. 1. In each row the images are $6 \mathrm{~s}$ apart. Between the last image of a row and the first image of the next row, there is a gap of $24 \mathrm{~s}$ while the camera was recording at other wavelengths. Five different colored fiducial marks have been placed at the same point in each image to help the eye follow temporal variations at particular locations.

$\log$ of the magnetic field magnitude. Green and black indicate regions of low magnetic-field strength where merging is expected. Last closed field lines are traced from each ionosphere and combined into a 3-D surface in GSE coordinates. The GSE $X$ axis is indicated by the + symbol. The intersection of the $X Y$ plane at $Z=6.9 R_{E}$ with the $X Z$ plane at $Y=-5.2 R_{E}$ pinpoints the location of Cluster.

Sets of "first open field lines" were traced after moving $1 \mathrm{~km}$ poleward of each last closed field line in the ionosphere. Four pairs of first open field lines appear in Fig. 4a. Lines r, t, $\mathrm{v}$, and $\mathrm{x}$ attach to the northern hemisphere ionosphere, while $\mathrm{s}, \mathrm{u}, \mathrm{w}$, and y map to the southern hemisphere ionosphere. Figures $4 \mathrm{~b}$ and $\mathrm{c}$ indicate ionospheric footprints of the selected open field lines mapped onto ionospheric potential patterns from the simulation in the northern and southern hemispheres, respectively. The apparent merging site for $r$, which traces through the approximate location of Cluster, and $\mathrm{s}$ is in the green region of the sash on the dawn flank near $X=4$ and $Y=-10 R_{E}$ (White et al., 1998). The southern-hemisphere footprint near 07:00 MLT is in region not covered by the allsky cameras.
The second pair of first open field lines, $t$ and $u$, originate from a merging site in the green region that extends upward from the dawn sash toward the cusp (highlighted by the black circle). While t maps near 09:00 MLT in the northern ionosphere, the footprint of its partner $\mathrm{u}$ is near magnetic noon in the southern ionosphere. Tracing nearby last closed field lines that connect to this region from the northern ionosphere results in a range of local times in the southern hemisphere. The variability is in part due to inaccuracies arising when the field line tracer must interpolate between grid points in regions of weak magnetic fields. Surface structure in the low field region with scale lengths less than $0.6 R_{E}$, represented by variations in shades of green, may be introduced spuriously by the tracer. On a larger scale, the ISM simulations show that in the region highlighted by a circle in Fig. 4a, low magnetic field regions activate and disappear repetitively every 3 to 4 min, suggesting that merging also does the same.

The third and fourth pairs of open field lines are representative of locations where merging is tied to the southern hemisphere sash and along the rim of the southernhemisphere cusp. The footprints in the southern hemisphere 

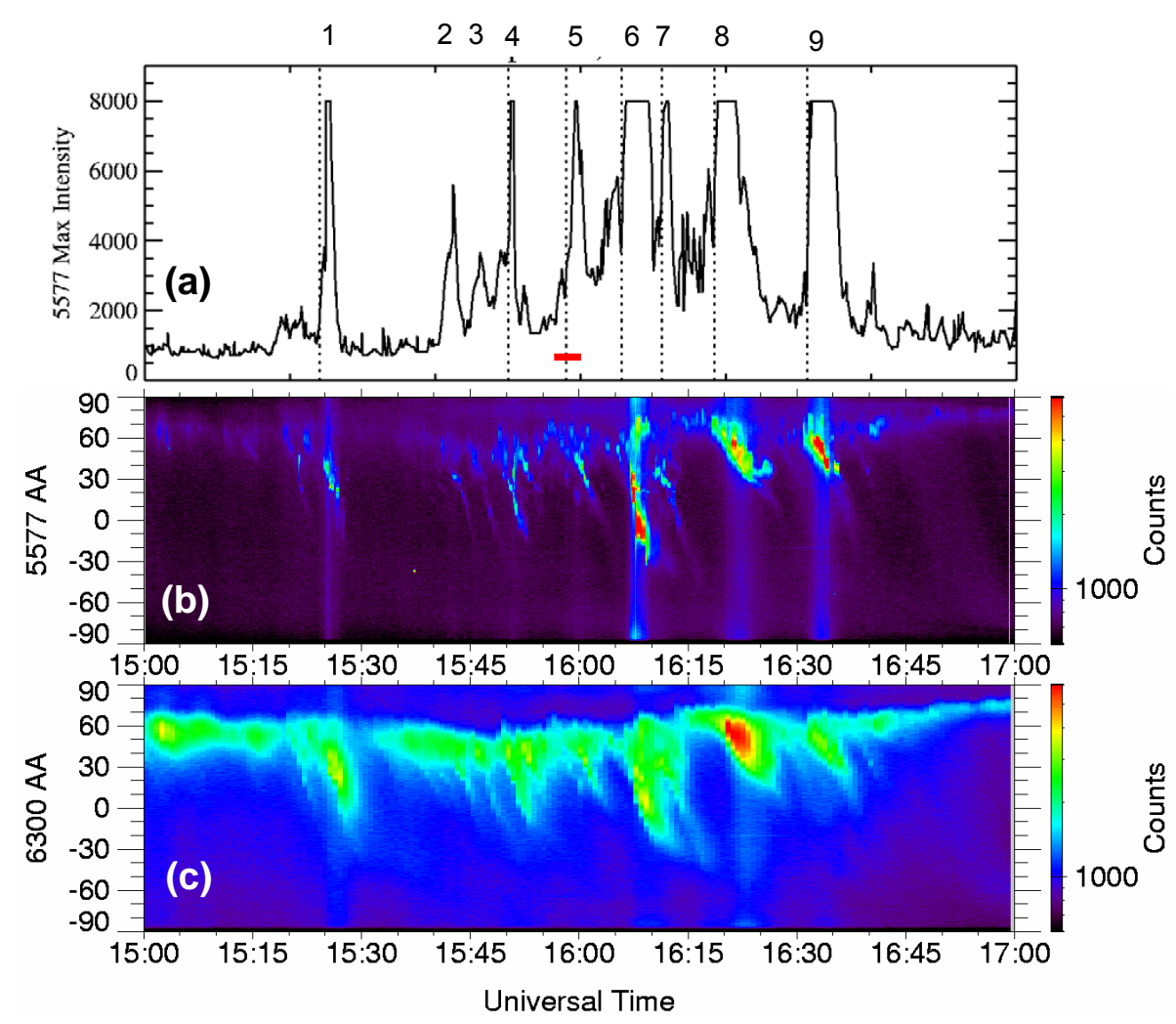

Fig. 6. (a) The maximum intensity of $557.7 \mathrm{~nm}$ emissions in each of the all-sky images versus UT for the two hour interval. (b, c) Keograms, or meridian-scan plot versus time, extracted from the $557.7 \mathrm{~nm}$ and $630.0 \mathrm{~nm}$ all-sky images. Emission intensities in counts are color-coded on a zenith-angle versus UT plot. The most poleward reaches of scans are at the bottoms of the keograms. The red bar in (a) marks the interval of images in Fig. 5. Note that each peak in the maximum intensity corresponds to a PMAF in the keograms.

range from near 13:00 to after 15:00 MLT but connect to locations on both sides of magnetic noon in the northern hemisphere.

These open field-line examples provide a large-scale context for understanding the $557.7 \mathrm{~nm}$ emissions found in the all-sky images that are presented below. The ISM simulation used constant inputs and did not try to follow the variability inherent in the data. However, the simulation illustrates how dipole tilt and $B_{X}$ strongly interact to affect magnetopause configurations.

\subsection{Temporal evolution of optical measurements on 29 April 2003}

Figure 5 shows a sequence of $557.7 \mathrm{~nm}$ images recorded at South Pole Station on 29 April 2003. Horizontal lines contain 4 images separated by $6 \mathrm{~s}$, with a $24 \mathrm{~s}$ gap between lines. Each image contains five colored fiducial marks located at the same places. By following activity near fiducials we see that at specific locations emissions may intensify briefly then dim and/or disappear. For instance, emissions begin at the pink fiducial in Fig. 5g, intensify in Figs. 5j, $\mathrm{k}$ and 1, and then weaken in the last-row images. Emissions near the yellow fiducial (separate from those of the blue fiducial) intensify in Fig. 5 g, becoming very bright in Figs. $5 \mathrm{~m}$, n, and o before dimming in Fig. 5p. Typically, the emissions intensify at a particular location for $30 \mathrm{~s}$ to several minutes, a phenomenology similar to that seen above Ny-Ålesund (Maynard et al., 2004). Multiple sites often activate simultaneously.

The trace in Fig. 6a indicates the maximum intensity (counts) of $557.7 \mathrm{~nm}$ emissions recorded in each individual image taken between 15:00 and 17:00 UT. The red bar shows the time interval of the acquisition of the images in Fig. 5. The large $557.7 \mathrm{~nm}$ intensification just before 16:00 UT reflects emissions near the yellow fiducial in Fig. 5. Figures $6 \mathrm{~b}$ and c, respectively, contain keograms of $557.7 \mathrm{~nm}$ and $630.0 \mathrm{~nm}$ intensities detected along the magnetic meridian at South Pole and plotted as functions of UT. The magnetic meridian connects the zenith at South Pole Station (center of each image) and the location of the magnetic pole. Both are marked by an $X$ in Fig. 1. The lowest magnetic latitude is at the top of these plots. The $630.0 \mathrm{~nm}$ emissions begin near the equatorward horizon then move to higher magnetic latitudes, typical of poleward moving auroral forms (PMAFs) (Sandholt et al., 1986). Major (and most minor) PMAF events are associated with intensifications of 557.7 

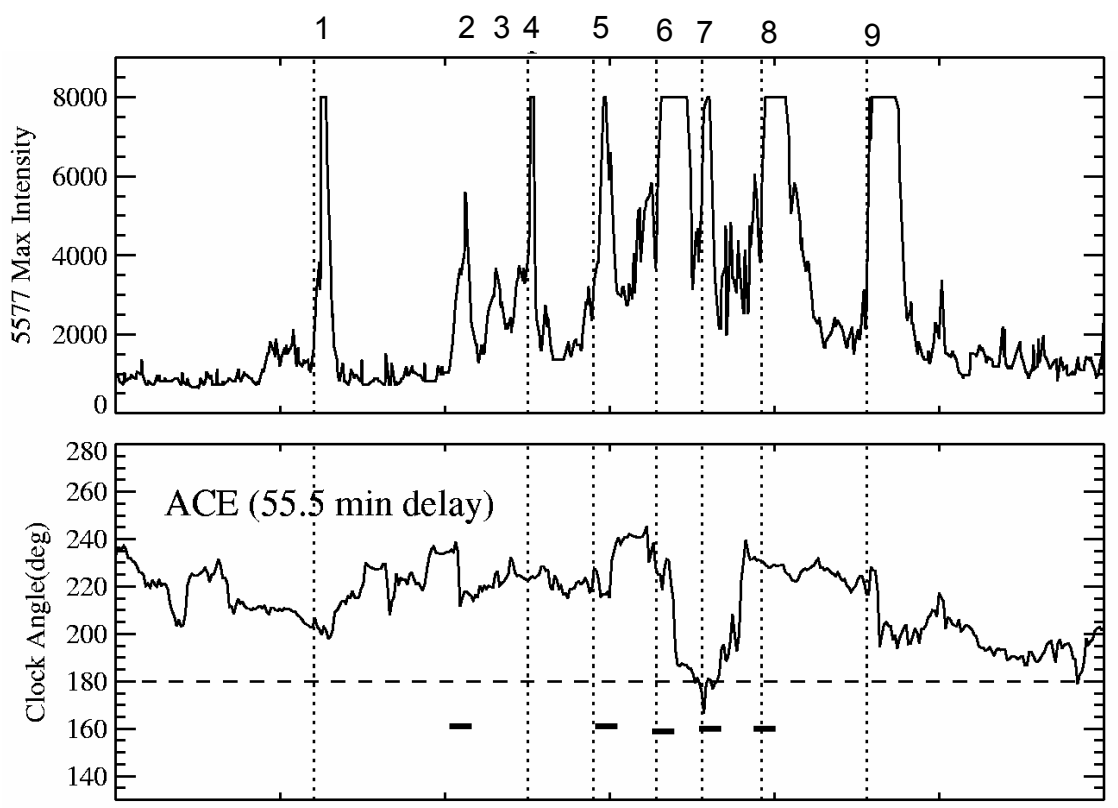

\section{(a)}

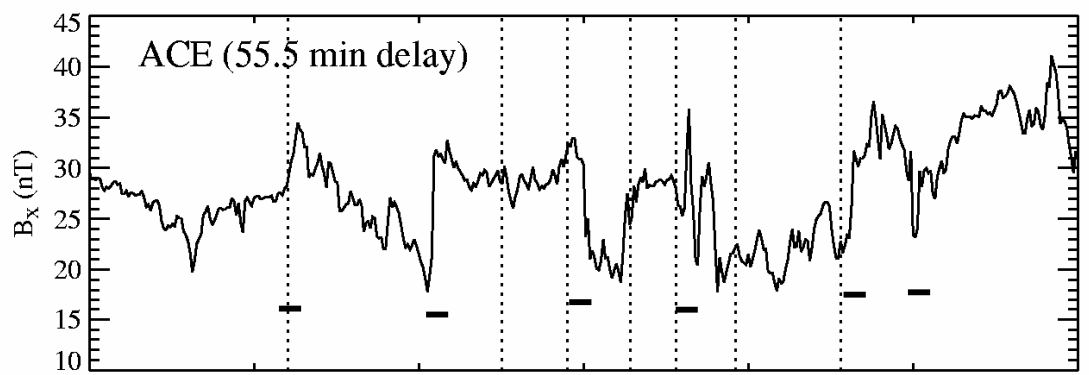

(c)

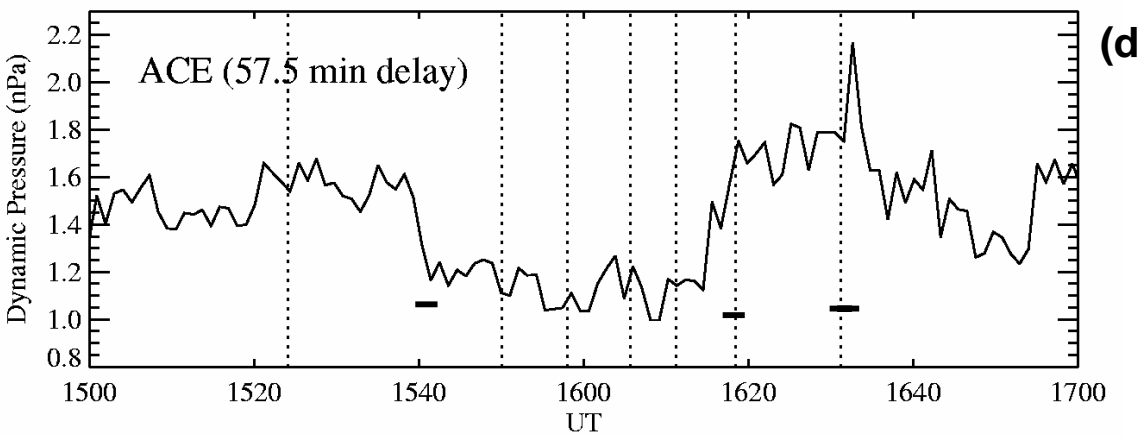

(b)

(d)

Fig. 7. Comparison of the maximum intensities (from Fig. 6) with possible solar wind drivers. An additional 2 min lag was included in the dynamic pressure plot (d) since its interaction occur at sub-cusp latitudes on the magnetopause rather than near the rim of the northern hemisphere cusp where the IMF makes first contact. Vertical dotted lines mark the beginning of each major PMAF. Black bars note changes in the solar wind parameters that may have triggered or influenced the PMAF.

emissions equatorward of South Pole and peak in the maximum amplitude (Fig. 6a). The times of maximum intensity in Fig. 6a may differ slightly from those on the keogram. This is because keograms only record intensities along the magnetic meridian, while emission maxima in Fig. 6a refer to positions anywhere within the all-sky imagers field of view. Also, the brightest emissions in the keogram have weaker features at all points along the meridian. This is an instrumental effect that raises background levels at all points in the all-sky image and may be disregarded.

The many intensity peaks in Fig. 6a leads us to ask what fraction of them is directly driven by solar wind parameters as opposed to internal magnetospheric processes. Results reported by Maynard et al. (2004) suggest that changes in $\theta$ cause variations in the intensities and locations of $557.7 \mathrm{~nm}$ emissions. Changes in IMF $B_{X}$ and $P_{S W}$ are also potential 

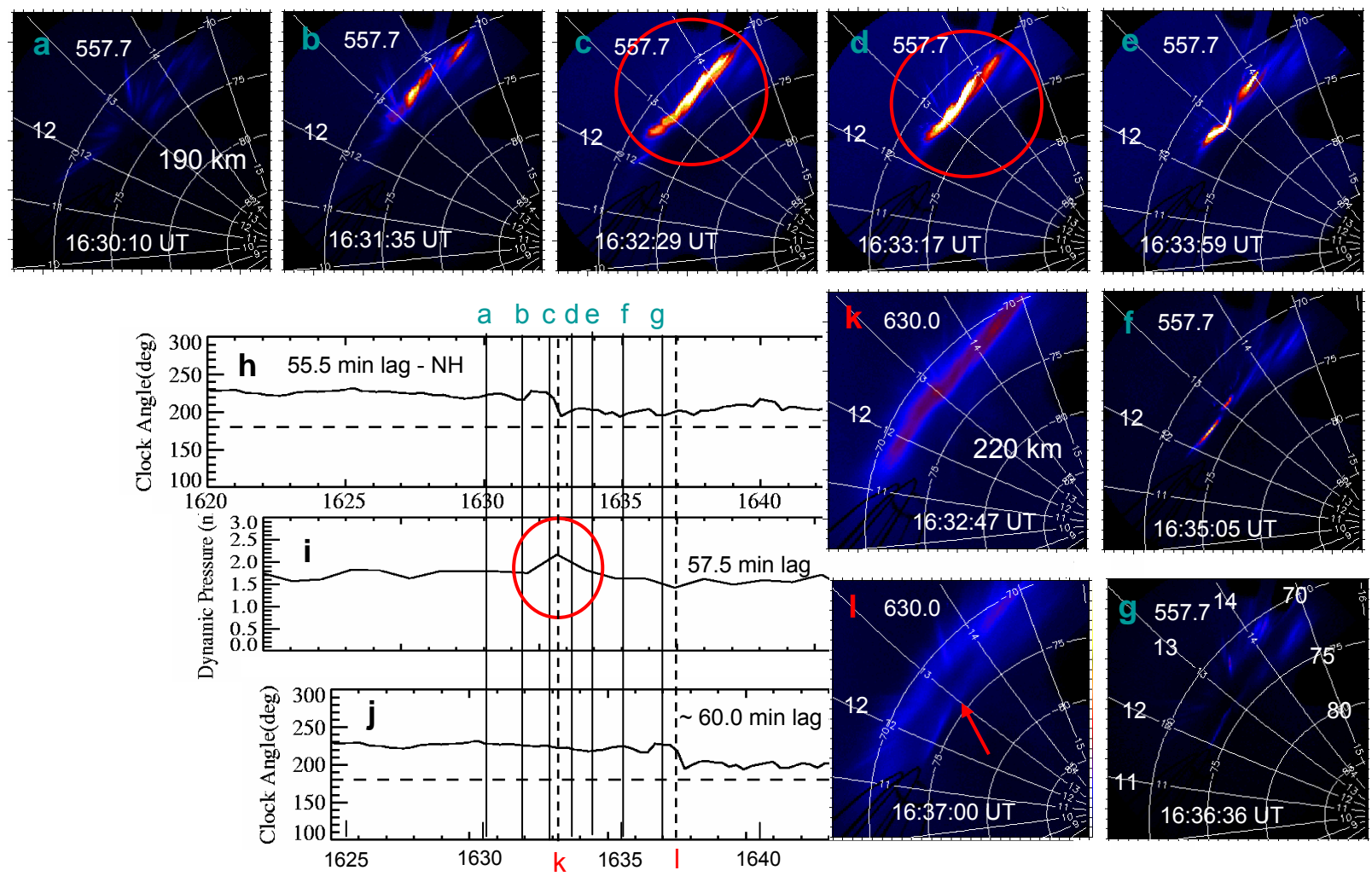

\section{Response to pressure change}

\section{South Pole April 29, 2003}

Fig. 8. (a-g) Selected $557.7 \mathrm{~nm}$ all-sky images near 16:32 UT when an impulsive pressure increase impacted the post noon dayside magnetopause. (h) The clock angle of the IMF measured by ACE with the lag determined for the northern hemisphere. (i) The ACE dynamic pressure lagged by $57.7 \mathrm{~min}$. (j) The IMF clock angle lagged by $60.5 \mathrm{~min}$, which is the appropriate lag for high latitude merging in the southern hemisphere. The red circles highlight the response to the pressure impulse. (k-l) $630.0 \mathrm{~nm}$ all-sky images at the peak of the disturbance and $\approx 4$ min later to show the poleward progression of the emissions.

sources of $557.7 \mathrm{~nm}$ variability. IMF $B_{X}$ variations alter the tilts of interplanetary electric field phase planes approaching the bow shock (Maynard et al., 2001b; Weimer et al., 2002) and thereby affect the times of interactions between the IMF and the magnetosphere in the two hemispheres. They also compress or expand the magnetic flux presented to a merging site (see Fig. 3). When IMF $B_{X}>0$ and $B_{Z}<0$, first interactions with the magnetosphere are favored in the northern hemisphere. Increasing the flux presented to a neutral line increases the rate of merging and enhances the creation of energetic electron tails responsible for $557.7 \mathrm{~nm}$ cusp emissions (Maynard et al., 2005). Thus, we expect that increasing (decreasing) IMF $B_{X}$ increases the merging rate in the northern (southern) hemisphere and thereby affects the South Pole all-sky images of the prenoon (postnoon) cusp. Solar wind pressure variations should also modulate access of magnetic flux to merging sites.
Figure 7 compares maximum intensities found in the allsky images (Fig. 6a) with variations of $\theta$, IMF $B_{X}$ and $P_{S W}$. ACE magnetic field data were lagged $55.5 \mathrm{~min}$, commensurate with the lag applied to Cluster near the northern hemisphere cusp (Fig. 2). On the other hand, $P_{S W}$ variations were lagged by $57.5 \mathrm{~min}$. Longer lags are needed for $P_{S W}$ effects to impact the subsolar magnetosphere and to reach expected post-noon southern hemisphere merging sites, because of the positive $B_{X}$ and the resulted tilted phase plane (Maynard et al., 2001b; Weimer et al., 2002). Black bars superposed onto the various interplanetary parameter plots (Figs. 6b-d) indicate possible associations with $557.7 \mathrm{~nm}$ intensifications (Fig. 7a). Note that some intensity peaks appear to have multiple possible associations with varying solar wind parameters. Detailed comparisons below address association/causality and timing issues. We use Peak 9 to illustrate responses to dynamic pressure changes; Peaks 1 and 2 to show $B_{X}$ effects; Peaks 6 and 7 help unravel responses 
to clock angle changes when multiple drivers act together. In the discussion we consider Peak 8 to point out similarities and differences in responses between a step function in pressure and the pressure impulse that caused Peak 9.

\subsection{Examples connecting IMF and solar wind variations with PMAFs}

\subsubsection{Peak 9: pressure pulse response}

A solar wind dynamic pressure spike (Fig. 7d) is clearly associated with the emission intensity peak observed near 16:32 UT (last peak in Fig. 7a). Figures 8a-g present seven $557.7 \mathrm{~nm}$ images surrounding the optical peak. The clock angle measured by ACE, lagged by $55.5 \mathrm{~min}$, is shown in Fig. 8h. This lag is applicable to Cluster magnetometer data for the interval and approximate interaction times in the northern hemisphere. Figure 8i depicts the ACE dynamic pressure lagged by $57.5 \mathrm{~min}$, while Fig. 8 j shows the clock-angle lagged by $60.0 \mathrm{~min}$ for southern-hemisphere interactions (and for analyzing emission peaks presented below). A pressure lag of $58 \mathrm{~min}$ is between the two extremes and should represent the maximum increase at the subsolar magnetopause. Since the IMF is in a spiral orientation, the pressure change should first impact the magnetopause postnoon. Vertical lines mark the times of the optical images (in Fig. 8). As the pressure increased, $557.7 \mathrm{~nm}$ emissions shown in Figs. 8c and d intensified over almost two hours of MLT. Comparing to Fig. 4c, we see that the emissions map near field line $\mathrm{w}$ and extend toward field line y. The northern hemisphere mappings of the newly opened field lines from the southern hemisphere merging site (lines $\mathrm{x}$ and $\mathrm{v}$ in Fig. 4b) extend from 11:00 to 13:00 MLT. Note that the $\theta$ applicable to the southern hemisphere did not change in this interval (Fig. 8j) in ways that would have affected southern hemisphere merging and/or post-noon optical emissions. Consistent with simulated field lines t, r, s, and u (Fig. 4) and experimental results of Maynard et al. (2001b, 2004), we expect that with the prevailing IMF $B_{Y}<0$, northern hemisphere merging sites were located well before local noon. They should map to the southern hemisphere ionosphere near or before 12:30 MLT. The only near-noon increase in $557.7 \mathrm{~nm}$ emissions occurred at 16:35 UT near 12:30 MLT (Fig. 8f), well after the optical and pressure enhancements. The $B_{X}$ increase (Fig. 7c) at the time of Peak 9 was offset by an increase in the magnitude of negative $B_{Z}$ (see Fig. 2). Thus, the possible flux compression illustrated in the cartoon of Fig. 3 and discussed below would not be significant. The magnitude of $\theta$ did change near 16:32 UT using the $55.5 \mathrm{~min}$ lag (Fig. 8h), appropriate for increased emissions conjugate to pre-noon northern hemisphere merging sites. However, there were no observed significant changes in optical emissions in the pre-noon southern hemisphere sector, and prenoon merging in the northern hemisphere can not account for the emission increases observed after 13:00 MLT in the southern hemisphere (see Fig. 4). Using a longer lag applicable to southern hemisphere merging sites (Fig. 8j), there were no changes in clock angle or $B_{X}$ near 16:32 UT when emissions increased. This isolates the cause of the enhanced post-noon emissions in Figs. 8c and d to the 15 to 20 percent increase in $P_{S W}$.

Figures $8 \mathrm{k}$ and 1 present $630.0 \mathrm{~nm}$ all-sky images projected to $220 \mathrm{~km}$ for times near and four minutes after the pressure pulse. The higher projection altitude is necessary because the lower energy electrons producing $630.0 \mathrm{~nm}$ emissions cannot penetrate deeply into the ionosphere to deposit energy. A poleward movement of the emissions during the interval is evident from the position of the poleward edge (red arrow in Fig. 81). This poleward movement occurred over a broad swath of magnetic local times, as did the original 557.7 and $630.0 \mathrm{~nm}$ enhancement. We return to this point in the discussion.

\subsubsection{Peaks 1 and 2: responses to IMF $B_{X}$ changes}

Figure 9 presents magnetic field and optical measurements acquired between 15:20 and 15:50 UT in order to illustrate effects associated with IMF $B_{X}$ changes. No significant changes in $\theta$ or $P_{S W}$ (Figs. $7 \mathrm{~b}$ and d) occurred at the first $557.7 \mathrm{~nm}$ emission enhancement in this interval (peak 1 in Fig. 7a). A large drop in dynamic pressure (Fig. 7d) and a clock angle decrease occurred at the second emission enhancement (peak 2 in Fig. 7a). Figures 9a-d show $557.7 \mathrm{~nm}$ emissions observed near 15:25 UT (peak 1 in Figs. 5a and 7a). Figures $9 \mathrm{e}$ and g present the 55.5-min lagged clock angle and $B_{X}$ measured by ACE. Figure $9 \mathrm{f}$ plots $B_{Y}$ measured by Cluster 1 (blue) and 4 (red). As noted above, changes in $B_{Y}$ provide the principal signature of crossing the magnetopause current layer. Cluster 4 led the constellation and crossed the magnetopause first, initially penetrating to the magnetosheath side of the current layer at 15:22 UT, evidenced by the brief reversal in $B_{Y}$ and exiting it near 15:30 UT. Cluster 1 crossed the current layer at 15:37 UT. Cluster 1 and 4 measurements of $|\mathbf{B}|$ are given in Fig. 9h. The discussion of Fig. 3 indicated our expectation for an increase in $B_{X}$ to enhance $|\mathbf{B}|$ and the flux presented to the merging line. Two significant increases in the lagged IMF $B_{X}$ are highlighted by the vertical lines in Figs. 9e-h. Figures 9a-d show increased $557.7 \mathrm{~nm}$ emissions at the time of the first of these changes. The red circle in Fig. $9 \mathrm{~b}$ highlights an emission increase that occurred near MLT noon. Because Cluster 4 was in the current layer and Cluster 1 had not reached it, no variation in the magnitude $B$ can be observed for this first event. Figure 4 suggests that newly opened field lines $t$ and $u$ respectively map from northern high-latitude merging sites to near 09:00 and 12:00 MLT in the northern and southern ionospheres. No significant emissions appeared near 13:00 MLT, as expected if merging occurred in the local southern hemisphere during this period of IMF $B_{Y}<0$. Finally, we note that the location of Cluster (field lines $r$ and $s$ in Fig. 4) maps to 


\section{$B_{X}$ change}

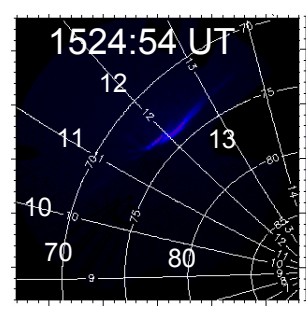

(a)

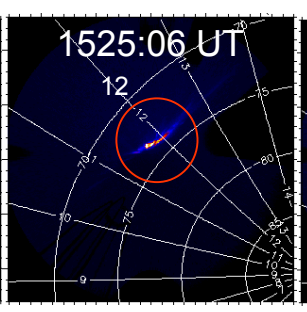

(b)

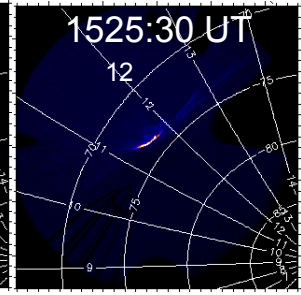

(c)

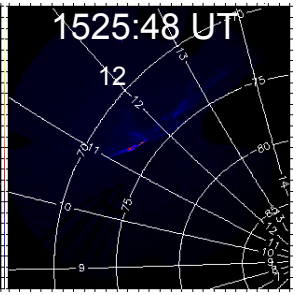

(d)

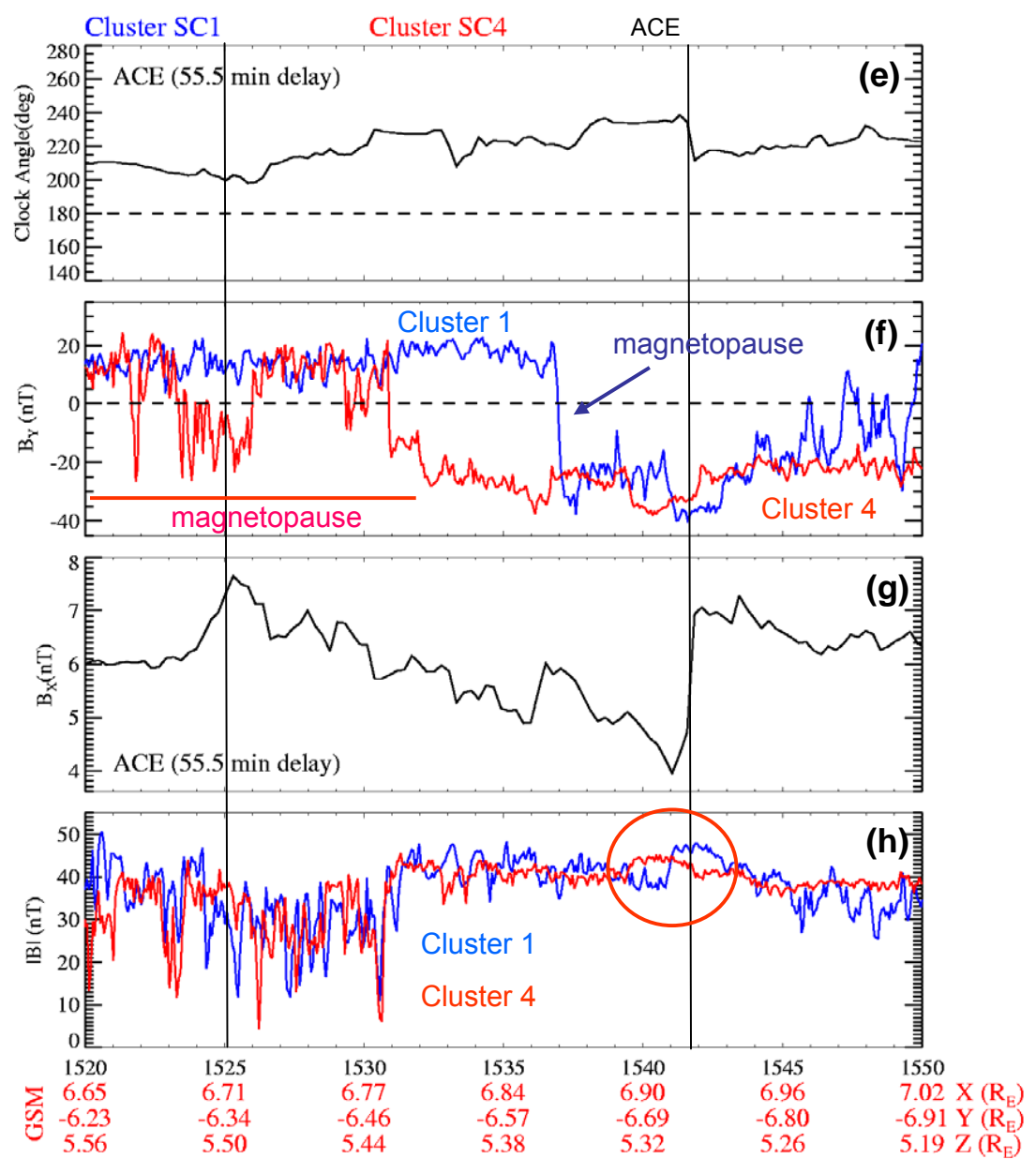

Fig. 9. Responses to increases in $B_{X}$. (a-d) $557.7 \mathrm{~nm}$ all-sky images near the period the increase in emissions at 15:25 UT. In data traces (e-h) the colors black, blue and red denote data source as ACE, Cluster 1 and Cluster 4, respectively. The four line trace panels show the clock angle, $B_{Y}, B_{X}$, and the magnitude of $B$. The left vertical line notes the increase in $B_{X}$ at the same time as the emission increase in panel (b). The right vertical line highlights the large increase in IMF $B_{X}$. At this time (panel h) an increase in $B$ is seen first by Cluster 4 and then by Cluster 1, which is closer to the magnetopause.

earlier local times than those covered by the all-sky imagers. However, the increase in $|\mathbf{B}|$ due to $B_{X}$ changes are expected over a range of MLT. Field lines $t$ and $u$ are representative of the brightest emissions in the all-sky image and suggest the location of enhanced merging.
At the time of the second vertical line in Figs. 9e-h (corresponding to the second $557.7 \mathrm{~nm}$ peak in Fig. 6a), both Cluster 1 and Cluster 4 were in the magnetosheath and the change in IMF $B_{X}$ appeared much sharper. The orange circle in Fig. 9h highlights increases in $|\mathbf{B}|$ seen by both Cluster 1 

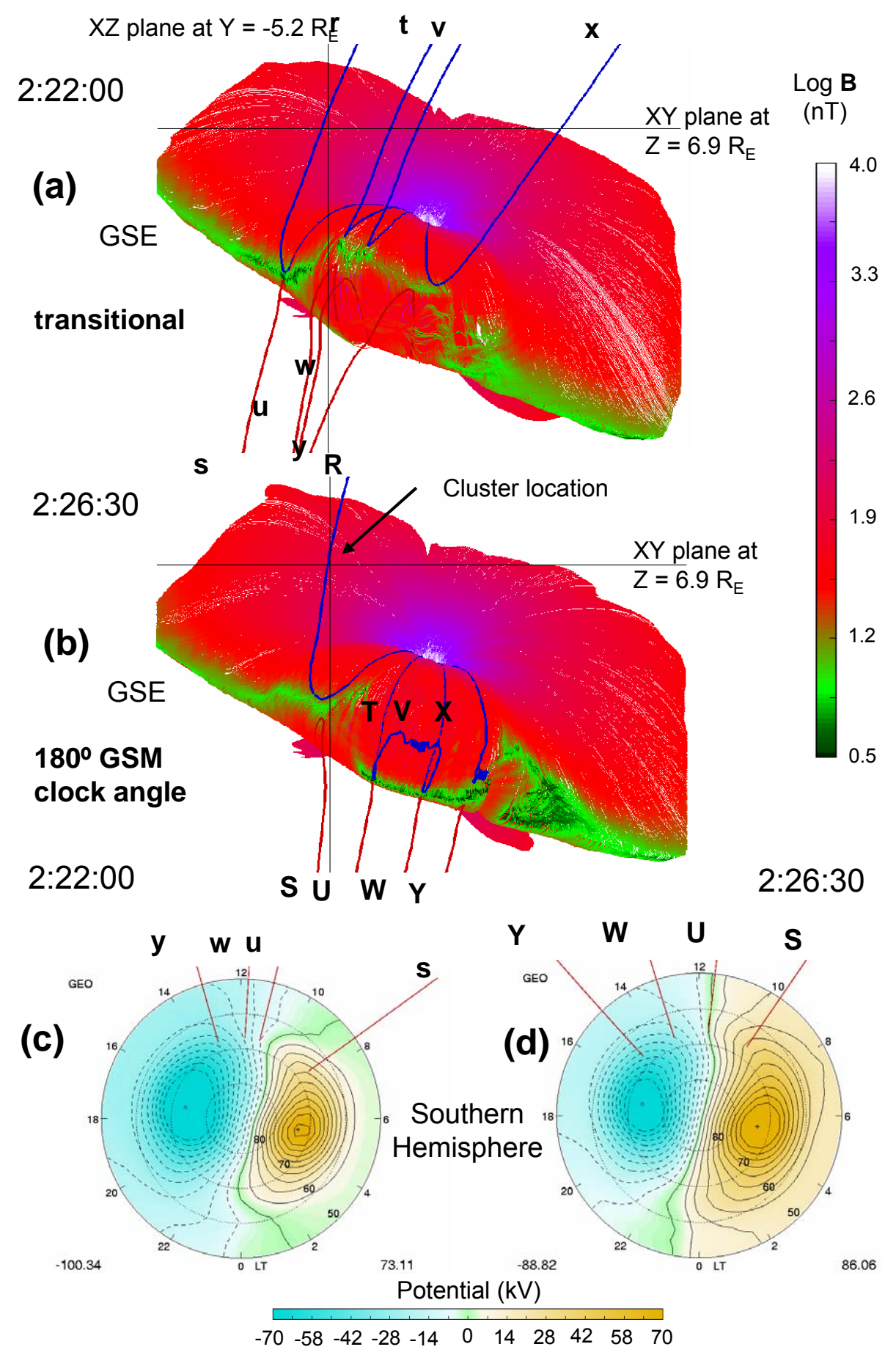

Fig. 10. Results from an ISM simulation with input conditions during and after the transition to $180^{\circ}$ clock angle. The format is similar to Fig. 4. (a) and (b) The 3-dimensional magnetopause as defined by the sets of last closed field lines mapped from the northern and southern hemisphere ionospheres in GSE coordinates. The field lines are colored with the log of the magnitude of the magnetic field. Regions of low magnetic fields (green and black) on the magnetopause surface are proxies for possible merging locations. Four pairs of first open field lines, labeled $\mathrm{r}$ through y for the transition time and $\mathrm{R}$ through $\mathrm{Y}$ for the time after the transition, highlight regions of importance in interpreting the $557.7 \mathrm{~nm}$ emissions (see text). The foot-points of the field lines in the southern hemisphere ionosphere for each case are shown in (c) and (d) with the respective potential patterns as background. 
Clock Angle Change
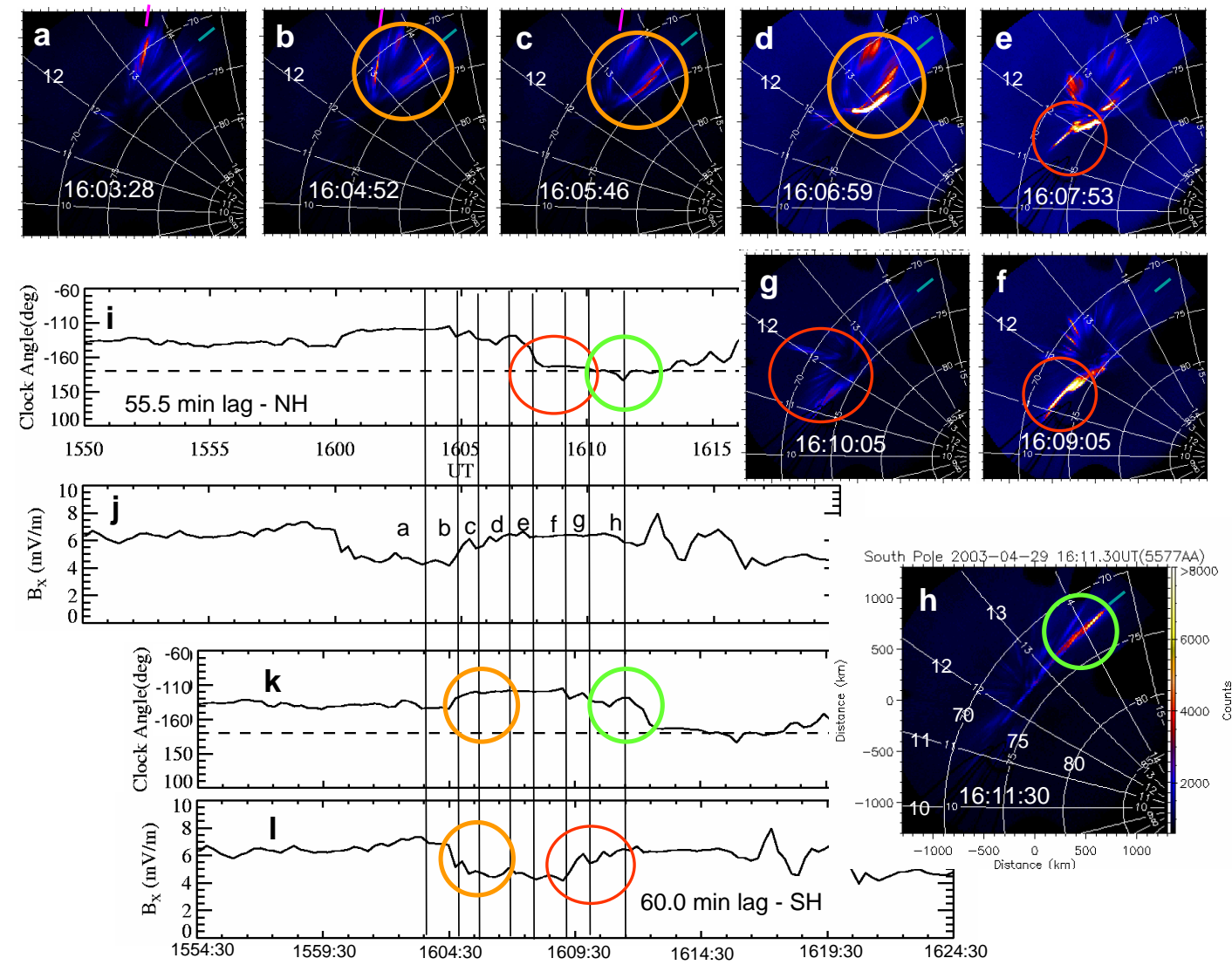

Fig. 11. Responses to clock angle changes. (a-h) $557.7 \mathrm{~nm}$ all-sky images at selected times noted by the vertical lines in the data trace plots (i-l). The clock angle and $B_{X}$ plots are shown using the measured lag for the northern hemisphere and the deduced lag for the southern hemisphere. To simplify references to the simulations we let clock angles assume negative values for $\theta>180^{\circ}$. Circles highlight changes in the IMF and apparent optical responses.

and 4. Located farther from the magnetopause, Cluster 4 saw the increase before Cluster 1. The Cluster 2 and 3 spacecraft were still on the magnetospheric side of the current layer and detected no flux increase. The enhanced magnetic flux presented to northern hemisphere merging sites due to the large positive change in $B_{X}$ appears to be as anticipated in Fig. 3. However, no clear optical signature near MLT noon was associated with this $B_{X}$ change. Figure $6 \mathrm{a}$ shows that a relatively weak $557.7 \mathrm{~nm}$ emission peak was observed 15:42 UT, but it was located near 13:00 MLT. Note in Fig. 7 that variations in $\theta$ and $P_{S W}$ accompanied this change in IMF $B_{X}$. The observed $P_{S W}$ decrease should tend to have the opposite effect to the $B_{X}$ increase on the strength of the electron tails for northern hemisphere merging, complicating conclusions about whether enhanced emissions should be observed.

\subsubsection{Peaks 6 and 7: responses to clock angle changes}

Before examining effects of clock angle changes on optical signatures we consider results of the second part of the ISM simulation (see Table 1 ) in which $\theta$ rotated to $180^{\circ}$ while $B_{X}$ remained constant. Figure 10 shows magnetopause configurations and southern-hemisphere potential patterns, in a format similar to Fig. 4. Simulation results depicted in Figs. 10a and $\mathrm{c}$ are appropriate for configurations achieved while in transition. Figures $10 \mathrm{~b}$ and d represent conditions after the new clock angle had affected the whole dayside magnetopause.

Like Fig. 4, four pairs of first-open field-lines are shown for two simulation times ( $\mathrm{r}-\mathrm{y}$ and $\mathrm{R}-\mathrm{Y}$ ). Note that newly open southern-hemisphere field lines $\mathrm{s}, \mathrm{u}, \mathrm{w}$, and y rotate from $45^{\circ}$ in Fig. 4a to the nearly vertical field lines S, U, W, and $\mathrm{Y}$ in Fig. 10b. The orientation of corresponding field lines in Fig. 10a lie in between, with y more like those in Fig. 4a and $\mathrm{u}$ and $\mathrm{w}$ more like those in Fig. 10b. Field lines $t$ and $v$ from near 09:00 MLT map to transient prenoon green regions on the magnetopause just north of the equator. Their southern hemisphere counterparts are between 11:00 and 12:00 MLT. Note that in the transitional period Cluster monitored field lines (s and $\mathrm{S}$ ) that mapped to after 
08:00 MLT in the southern hemisphere, versus mapping to 07:00 MLT in Fig. 4c.

Figure $10 \mathrm{~b}$ suggests that merging was strong across the magnetospheric noon sector along the equatorward rim of the southern-hemisphere cusp. Field lines U, W, and Y map to between 11:00 and 15:00 MLT in the ionosphere (Fig. 10d). Cluster data would then be associated with field lines (S) that mapped to near 09:00 MLT in the southern hemisphere. Note that tracings of field lines $\mathrm{T}, \mathrm{V}$, and $\mathrm{X}$ are inherently uncertain in the low field regions close to merging sites near the subsolar magnetopause.

Figure 11 shows a case in which clock-angle changes coincided with $B_{X}$ decreases. This example corresponds to intensity peaks 6 and 7 in Fig. 6a. Here we must allow different lag times for solar wind packets to reach northern and southern hemisphere merging sites. Figures 11a$\mathrm{h}$ show $557.7 \mathrm{~nm}$ all-sky images at times denoted by the vertical lines in the line plots of Figs. 11i-l. Figures 11i and $\mathrm{j}$ show the clock angle and $B_{X}$, with the $55.5 \mathrm{~min}$ lag appropriate for northern-hemisphere merging. The dashed line in Fig. $11 \mathrm{i}$ marks $\theta \approx 180^{\circ}$, (i.e. $B_{Y} \approx 0$ ). Figures $11 \mathrm{k}$ and 1 show the clock angle and $B_{X}$ with the $60.5 \mathrm{~min}$ lag that provides the closest match of changes in the solar wind driving parameters to the southern-hemisphere auroral features observed between 13:00 and 14:00 MLT during this and subsequent UT intervals. We expect that the shift in clock angle away from $-180^{\circ}$ to smaller negative values (orange circle in Fig. 11k) should coincide with the southern hemisphere merging site moving to later magnetic local times. Prior to the clock-angle change, the strongest emissions came from near 13:30 MLT, corresponding to the pink fiducial (Fig. 11a). In Figs. 11b and c the strongest emissions came from locations near 14:00 MLT and the higher latitudes marked by the green fiducial. Meanwhile emissions near the pink fiducial nearly vanished. This shift is similar to that observed post-noon in the northern hemisphere at NyÅlesund for positive clock angles (Maynard et al., 2004). At about the same time $B_{X}$ was decreasing, possibly enhancing the magnetic flux presented to southern-hemisphere merging sites. The cumulative effects of these two potential drivers are seen in Fig. 11d as an intensifying, westward-moving auroral patch at higher latitudes (the high latitude branch of the emissions will be addressed in Sect. 4.3), with other emissions having moved to near 14:00 MLT.

Applying the lag determined from Cluster data in the northern hemisphere, we see that the IMF clock angle rapidly shifted toward $180^{\circ}$ at 16:08 UT (red circle in Fig. 11i). Responses in the South Pole all-sky images appear as strong emissions in the noon and pre-noon MLT sectors (red circles in Figs. 11e and f). Lines $u$ and $w$ in Fig. 10a and $c$ and line $\mathrm{U}$ in Figs. 10b and $\mathrm{d}$ are representative of the mappings. Pre-noon emissions continued, albeit with weakened intensities, past 16:10 UT (Fig. 11g). At this time weak, structured emissions extended from about 11:00 to 14:00 MLT. With the $\theta \approx 180^{\circ}$, merging should proceed near the magne- tospheric equatorial plane over a broad range of local times. The multiple rays at various local times in Fig. 11g suggest that merging did not occur uniformly at all local times, but was patchy across the dayside. Note also that, at the time of the emissions shown in Fig. 11g, IMF $B_{X}$ was increasing (Fig. 111), thus decreasing the rate at which flux was presented to southern-hemisphere merging sites. Consistent with this observation, emissions were weaker in the postnoon than in the prenoon sector and less intense than those in previous images. At 16:11:30 UT (Fig. 11h) emissions in the 13:00-14:00 MLT sector (green circle) strengthened (second to last peak in Fig. 6a). This corresponds to the time when $\theta$ crossed to positive values (Fig. 11i), thus favoring merging at postnoon sites in the northern hemisphere. With postnoon sites still active in the southern hemisphere, nearly all the emissions came from between 13:00 and 14:00 MLT. The brief intensification spanned one hour in MLT and reached to the location of the emissions highlighted by the green fiducial in Figs. 11b-d.

\subsection{Signatures of Northern-Hemisphere merging}

With $\theta \approx-135^{\circ}$ merging in the northern hemisphere should occur on the morning side of the cusp where the interplanetary and terrestrial fields are nearly anti-parallel (Crooker, 1979; Luhmann et al., 1984; Maynard et al., 2003). This is close to the location of Cluster. Energetic electron tails (keV energies), identified by Maynard et al. (2005) as signatures of active merging, appear in the Cluster measurements acquired on the magnetosheath side of the current layer. Figures 12a, $\mathrm{b}, \mathrm{c}, \mathrm{h}, \mathrm{i}$, and $\mathrm{j}$ show energy-versus-time spectrograms of parallel, perpendicular and anti-parallel electron fluxes sampled by Cluster 4 and Cluster 1, respectively. Figures 12d, e, f, and $\mathrm{g}$, respectively, show the three components and magnitude of proton velocities as measured by the CIS/CODEF sensor on Cluster 1. Cluster 1 initially crossed the magnetopause current layer at 15:37 UT, then returned to the vicinity of the current layer from 15:45 to 15:55 UT (Fig. 9). Energetic electron tails moving anti-parallel to $\mathbf{B}$ appear intermittently throughout the interval. Another episode of energetic electron tails occurred between 16:08 and 16:12 UT. Cluster 4 also encountered anti-parallel, electron-tail events near 15:37 UT, between 15:45 and 15:52 UT, and at 15:25 UT. Note that all of the enhanced electron flux events are unidirectional with no particles mirroring from the ionosphere. Field-line traces in Fig. 4 indicate that this is consistent with Cluster crossing outer-separatrices (Maynard et al., 2005) connected to active merging sites located equatorward and dawnward of the spacecraft.

Three ion-velocity peaks occurred between 15:46 and 15:52 UT (Fig. 12g). These enhancements were principally in the $+Y$ direction, indicating field-line motion across the cusp toward noon, as suggested by field line $r$ in Fig. 4. Another velocity peak, sampled near 16:11 UT, was principally in the $+Z$ and $-X$ directions, indicated by field line $\mathrm{R}$ in 
Fig. 10b. The lesser $Y$ component shift was negative. In the data presentation of Fig. 11, we showed that the applicable clock angle for northern hemisphere merging rotated from $145^{\circ}$ to near $-180^{\circ}$. The altered ion-velocity direction is, as expected, consequent to the clock-angle change.

To confirm our hypothesis that active merging was occurring on field lines intersecting Cluster locations, we performed a Walén test using ion velocities measured during the interval marked by the red bar in Fig. 12f. The Walén test is based on tangential momentum balance and is a property of rotational discontinuities associated with magnetic merging (see Sonnerup et al., 1981). Figure 13 plots the components of the magnetic field perturbation $\Delta \mathbf{B}$ versus $\Delta \mathbf{A}$, where $\mathbf{A}$ is defined by $(4 \pi \rho)^{1 / 2} \Delta \mathbf{V}$. We assume temperature isotropy of the plasma. Perturbations in the velocity and magnetic fields are obtained by subtracting average background values from the measured values. background values are calculated using running boxcar averages of width 1 min (15 points). Linear fits of unit slope that pass through the origin indicate the boundary was a rotational discontinuity. Results found in Fig. 13 are generally consistent with these expectations. Another successful Walén test was also performed on Cluster 4 measurements between 15:21 and 15:27 UT while the spacecraft was crossing the magnetopause current layer.

The active merging site near Cluster must produce electron tails moving along the inner separatrix connected to the southern hemisphere cusp. Therefore optical signatures produced by these electrons should be found in conjugate allsky images from South Pole station. Since the tail electrons move at speeds near $20000 \mathrm{~km} / \mathrm{s}$, their optical signatures in the southern ionosphere appear almost instantaneously. However, Fig. 4 shows that when the clock angle was near $145^{\circ}$, newly opened field lines that mapped near the location of Cluster came from merging sites on the magnetopause flank called the "sash" (White et al., 1998). Related optical emissions are excited at earlier local times than those covered by the South Pole all-sky camera. Electrons responsible for $557.7 \mathrm{~nm}$ emissions observable in South Pole all-sky images near noon and earlier local times originate at merging sites in the northern hemisphere located between 09:00 and 11:00 MLT (Fig. 4). Hence, Cluster measurements made with $\theta \approx-145^{\circ}$ provide information about when merging was enhanced in the northern hemisphere. However, Cluster electrons were not tied to optical emissions that were simultaneously observable at South Pole Station. Figures $10 \mathrm{~b}$ and d indicate that with $\theta \approx 180^{\circ}$ the conjugate merging site monitored by Cluster moves to later local times, but associated $557.7 \mathrm{~nm}$ emissions still lie outside the South Pole all-sky cameras field of view.

Figures 121-o show all-sky images acquired at four of the five times when Cluster observed ion-velocity peaks and energetic electron tails, keyed by vertical dashed lines. The first three events occurred while $\theta \sim 145^{\circ}$. Figure 121 corresponds to first intensity peak in Fig. 6a and is related to a noontime enhancement following a change in $B_{X}$ (cf. dis- cussion of Fig. 9). Figure 12m corresponds to the fourth intensity peak in Fig. 6. Figure 12n corresponds to a small enhancement in the maximum intensity shortly after the fourth peak in Fig. 6a. Figure 12o references a time when the clock angle appropriate for northern-hemisphere merging changed to $\sim 180^{\circ}$ (just before the second to last peak in Fig. 6). In Figs. 121 and $\mathrm{m}$ an emission enhancement appeared very close to 12:00 MLT (highlighted by a red circle). ISM simulations connect the observed auroral emissions to northern hemisphere merging sites (field lines $\mathrm{t}$ in Fig. 4, t in Fig. 10a, and $\mathrm{T}$ in Fig. 10b) but not to the newly opened magnetic field lines monitored by Cluster, where the velocity enhancements were observed. In Fig. 12o the emissions extend from about 10:00 to past 14:00 MLT. Simulation results in Fig. 10b (field line $S$ ) suggest that the emissions near 10:00 MLT in Fig. 12o are associated with, but not exactly at, the merging activity detected by Cluster.

\section{Discussion}

All but one of the observed PMAFs (FTEs) observed at South Pole Station during the selected the two-hour interval is demonstrably associated with changes in the solar wind that most likely initiated by them. Changes in $P_{S W}, B_{X}$ and/or $\theta$ are associated with each of the significant increases in the $557.7 \mathrm{~nm}$ emissions. The following subsections explore the impacts of the three solar-wind drivers on transient merging at the dayside magnetopause.

\subsection{Pressure-pulse responses}

Pressure pulses compress the dayside magnetosphere, locally for small and globally for large enhancements. For the magnetosphere to evolve from an initial to a final equilibrium configuration either the merging rate must increases to remove closed flux from the dayside, or closed field lines must be pushed aside and then pulled tailward by tangential viscous forces exerted along the flanks of the magnetopause. If the merging rate increases to accommodate the transfer of open flux from the day to the night side of the Earth, its effects should be manifest in the form of FTEs and PMAFs (Russell and Elphic, 1979; Sandholt et al., 1986) that couple to the ionosphere via characteristic field-aligned current signatures (Basinska et al., 1989). Alternatively, azimuthal gradients in the contact surface of pressure fronts with the magnetopause couple to the ionosphere via propagating pairs of field-aligned currents (Southwood and Kivelson, 1990; Sibeck, 1990). Under spiral-IMF conditions of 29 April 2003, pressure fronts first impact the magnetopause on the afternoon side, then move across the noon toward dawn (Sibeck, et al., 2003). Whether associated field-aligned currents result from merging or pressure sources may be difficult to discern initially. In both cases the principal FAC is upward, with downward return currents located to the side 


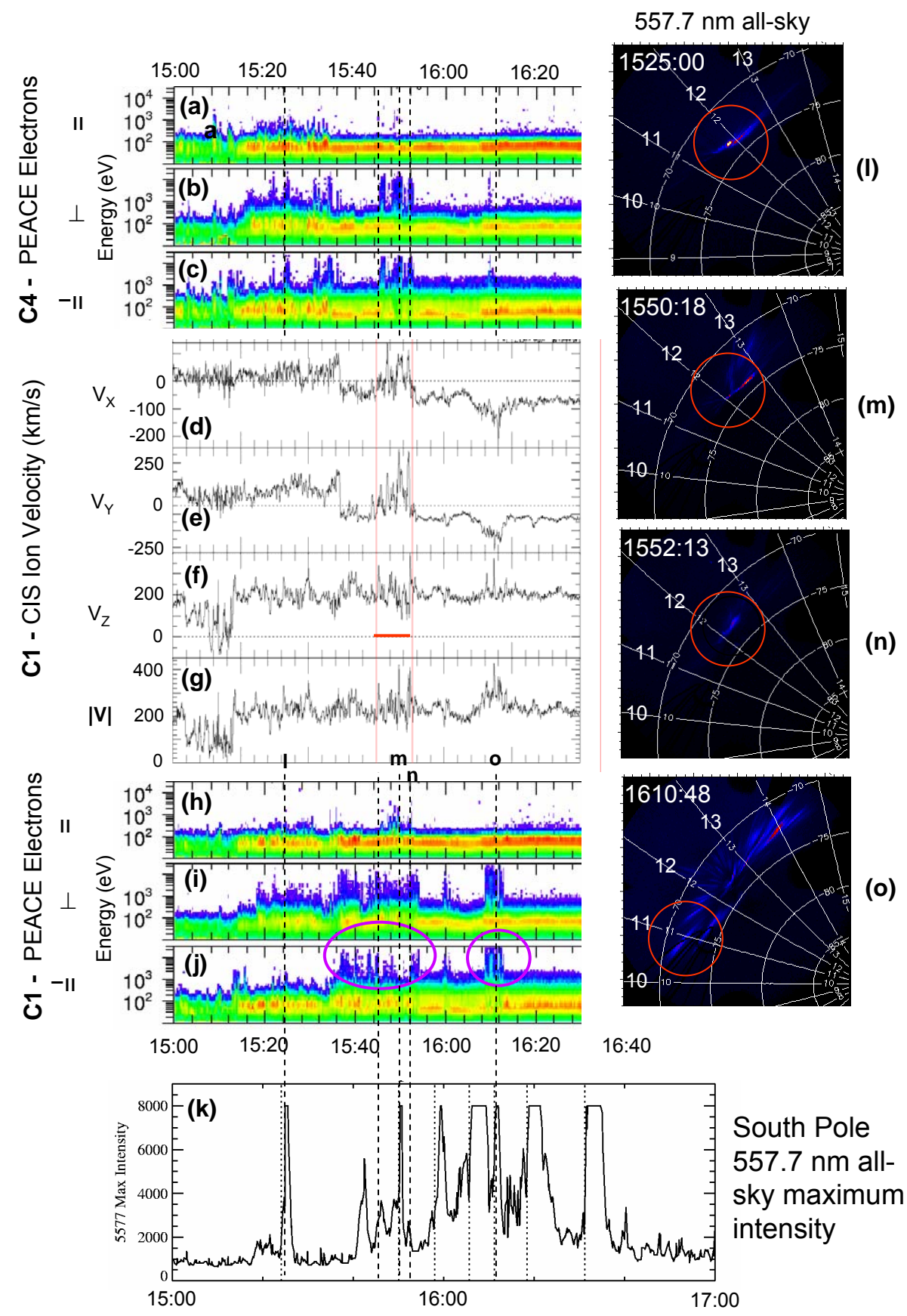

Fig. 12. Cluster 1 and 4 data compared with optics. (a-c) C4 PEACE electrons measured in directions parallel, perpendicular and antiparallel to B. (d-g) C1 CIS proton velocity components and magnitude measured by the CODIF sensor in GSE coordinates. (h-j) C4 PEACE electrons in directions parallel, perpendicular, and anti-parallel to B. The maxima in $557.7 \mathrm{~nm}$ emissions from South Pole (from Fig. 5) are shown for comparison. Dashed lines in panels $(\mathrm{a}-\mathrm{c})$ and $(\mathrm{h}-\mathrm{k})$ mark CIS velocity increases that have corresponding increases in the South Pole maximum intensities. Also, mono-directional electron tails appear in panels (c) and/or (j) at these times. (l-o) 557.7 nm images at the four selected times noted by dashed lines. Red vertical lines in panels $(\mathrm{d}-\mathrm{g})$ designate the interval of a successful Walén test (Fig. 13).

and in the wake (e.g. Sibeck et al., 2003; Denig et al., 1993). Negative IMF $B_{Y}$ causes newly merged flux to propagate toward dawn in the southern hemisphere, as do perturbations on the magnetopause from the pressure front as it expands across the dayside. However, if viscous forces dominate, subsequent plasma convection should continue toward dawn 

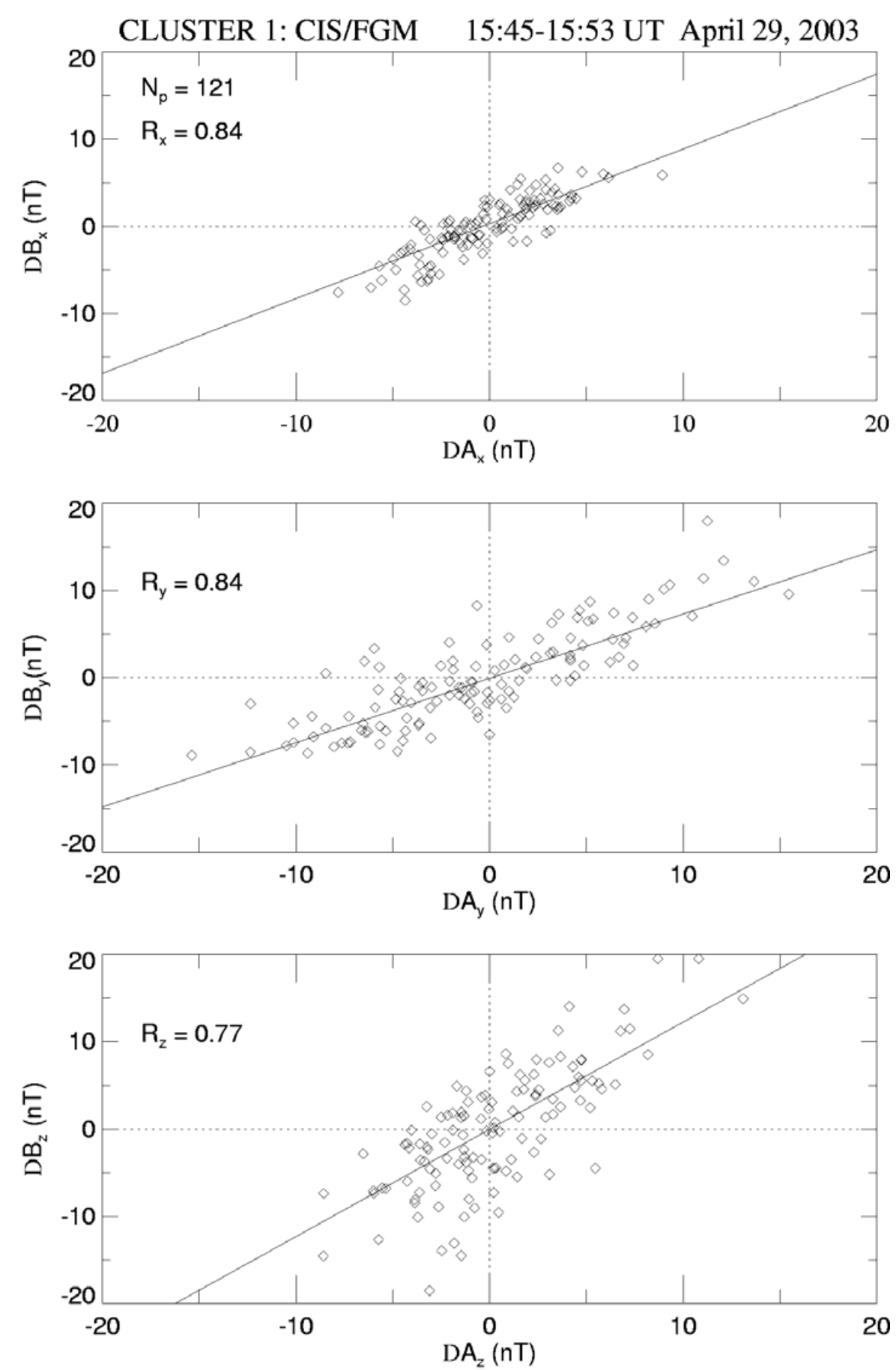

Fig. 13. Scatter plots checking the Walén relationship for the interval from 15:45 to 15:53 UT. Cluster 1 FGM and CIS data are shown for each of the components of the change in the magnetic field versus the change in the abscissa. The abscissa is defined as $\Delta A=(4 \pi \rho)^{1 / 2} \Delta V$. The slope of the linear regression is noted in each component plot.

and dusk along the open-closed boundary, away from the point of first impact. If on the other hand merging dominates, consequent FTEs and PMAFs should take more poleward paths through the region of open field lines.
The isolated pressure event at 16:32 UT (Fig. 8) was impulsive and less than $20 \%$ in amplitude. $P_{S W}$ rapidly increased then decreased in concert with a solar wind density fluctuation (Fig. 2e). The subsequent velocity decrease 
(Fig. 2f) helped to reduce pressure after the density peak. While the pulse amplitude was small, the optical responses in the ionosphere were significant. Bright emissions spanned the sky between 12:30 and 14:00 MLT along the equatorward rim of the cusp (Figs. 8b-e) throughout the pulse duration. Other types of interplanetary drivers produced multiple ray structures. Note that pressure decreases force the magnetosphere to inflate and thus decrease the merging rate.

If the sources of enhanced $557.7 \mathrm{~nm}$ emissions were electron tails emerging from a ctive merging sites, then the breadth in local time of the emissions indicates that increased merging occurred over a large swath along the magnetopause. The rate of flux transfer from the day to the night side can increase in two ways. Either the local merging rate or the length of the active merging line on the magnetopause grew. Both could cause enhanced emissions over a broad region. However, the lack of a change in $\theta$ applicable to southern-hemisphere postnoon merging sites or in $V_{S W}$ associated with the pressure increase suggests that the applicable interplanetary electric field was mostly unaltered. Thus, any local changes in the tangential electric field at the magnetopause resulted from distortions of the boundary. No responses were detected at prenoon local times, even several minutes after initial impact (Fig. 8g). The impulse only caused optical responses in the region of first contact.

Sandholt et al. (1994) observed that $630.0 \mathrm{~nm}$ auroral emissions in the cusp shift equatorward in response to pressure pulses and concluded that it was "likely that magnetopause reconnection was involved". Within the magnetosphere compressive waves launched by pressure pulses increase the magnitude of $\mathbf{B}$ (Southward and Kivelson, 1990). Korotova et al. (1999) used this feature to track the effects of pressure pulses through geosynchronous orbit. Thus, we expect that ground magnetometers should also detect $|\mathbf{B}|$ increases at high and mid latitudes. Figures $14 \mathrm{~b}-\mathrm{e}$ displays the three components and magnitude of $\mathbf{B}$ measured at South Pole. Also shown are traces of $P_{S W}$ measured by from ACE and Wind (Fig. 14g) and the maximum $557.7 \mathrm{~nm}$ intensities (Fig. 14a). An increase of $40 \mathrm{nT}$ in $\mathbf{B}$ as well as variations in all three components occurred at 16:32 UT (red bar). A smaller, but more impulsive, increase in $|\mathbf{B}|$ was seen pre noon MLT sector at Poste de la Baleine in Quebec (Fig. 14f). The magnetometer on GOES 6 (not shown) also detected an increase in $|\mathbf{B}|$. These variations are consistent with largescale changes in the magnitude of $\mathbf{B}$ expected from a pressure pulse interpretation. However, the increase in the magnitude of $\mathbf{B}$ does not address the question as to how the change was implemented. Our event is close to the smallest of the $40 \mathrm{nT}$ ground disturbance MIEs surveyed by Konik et al. (1994). They concluded that sporadic reconnection was involved in most of their events.

The strong $557.7 \mathrm{~nm}$-emission increase suggests that merging was the primary means for removing flux from the dayside and reconfiguring the magnetopause during this pressure pulse event. A PMAF was generated (last event in
Fig. 6) in which the $630.0 \mathrm{~nm}$ auroral form moved poleward (Figs. 8k and l) at all local times where strong increases in 557.7 emissions were seen. If merging were not involved in the reconfiguration, then the primary motion would have been azimuthal. Instead, the all-sky images of Fig. 81 show a band of $630.0 \mathrm{~nm}$ emissions (red arrow) with a width of over $2 \mathrm{~h}$, poleward of, and separated from the original location of the emissions (Fig. 8k). Intervening images followed this motion poleward. We conclude that the pressure pulse changed the magnetopause position via merging, and launched a PMAF. Recent complementary studies using Cluster, Image and SuperDARN data have also reached the conclusion that the reconnection rate is modulated by dynamic pressure changes (Bosqued et al., 2005; Cerisier et al., 2005).

A larger, and more spread out pressure response occurred just before 16:20 UT (green bar in Fig. 14g). This $P_{S W}$ event involved increases in both density and velocity, complicated by simultaneous changes in $B_{X}$ and $\theta$. The magnetometer at Poste de la Baleine detected a significant increase in $|\mathbf{B}|$; the increase at South Pole was smaller and wave-like.

Figures $14 \mathrm{~h}-1$ detail the optical responses to this increase that was more step like in character. In this case the $557.7 \mathrm{~nm}$ optical response began near 14:00 MLT then spread westward past magnetic noon, consistent with the picture of the pressure changes propagating along the magnetopause toward dawn (Sibeck et al. 2003; Korotova et al. 2004). $630.0 \mathrm{~nm}$ emissions (not shown) also spread westward and gradually poleward. The intensity of the structure may reflect contributions from both $B_{X}$ and $P_{S W}$ changes. Note, however, that emissions continued in the 13:00 to 14:00 MLT region as the front moved past noon. Increased emissions over a broad range of local time and subsequent poleward movement of the $630.0 \mathrm{~nm}$ emissions (PMAF) are consistent with a merging interpretation of the magnetopause reconfiguration required by the step-like change in $P_{S W}$.

In Fig. 14e the largest change in $|\mathbf{B}|$ at South Pole occurred near 16:08 UT, as $\theta$, applicable to the northern hemisphere, changed (Figs. 11e and f). This MIE was strongest in the transverse components while the change in the $Z$ component was bipolar. Magnetic variations are consistent with a localized, eastward current sheet in the ionosphere overhead that was moving poleward. We return to this event below.

\subsection{IMF $B_{X}$ changes}

IMF $B_{X}$ and the dipole-tilt angle affect the timing of merging-site activations and their locations. Maynard et al. (2001b) found that different lag times apply to effects observed in the pre- and post- noon ionosphere. They attributed the differences to the polarity of IMF $B_{X}$ prevailing at the time of the measurements. Phase planes of the interplanetary electric field (IEF) were tilted by the presence of $B_{X}$. Weimer et al. (2002) compared plasma and magnetic field data streams at the locations of four satellites in the solar 

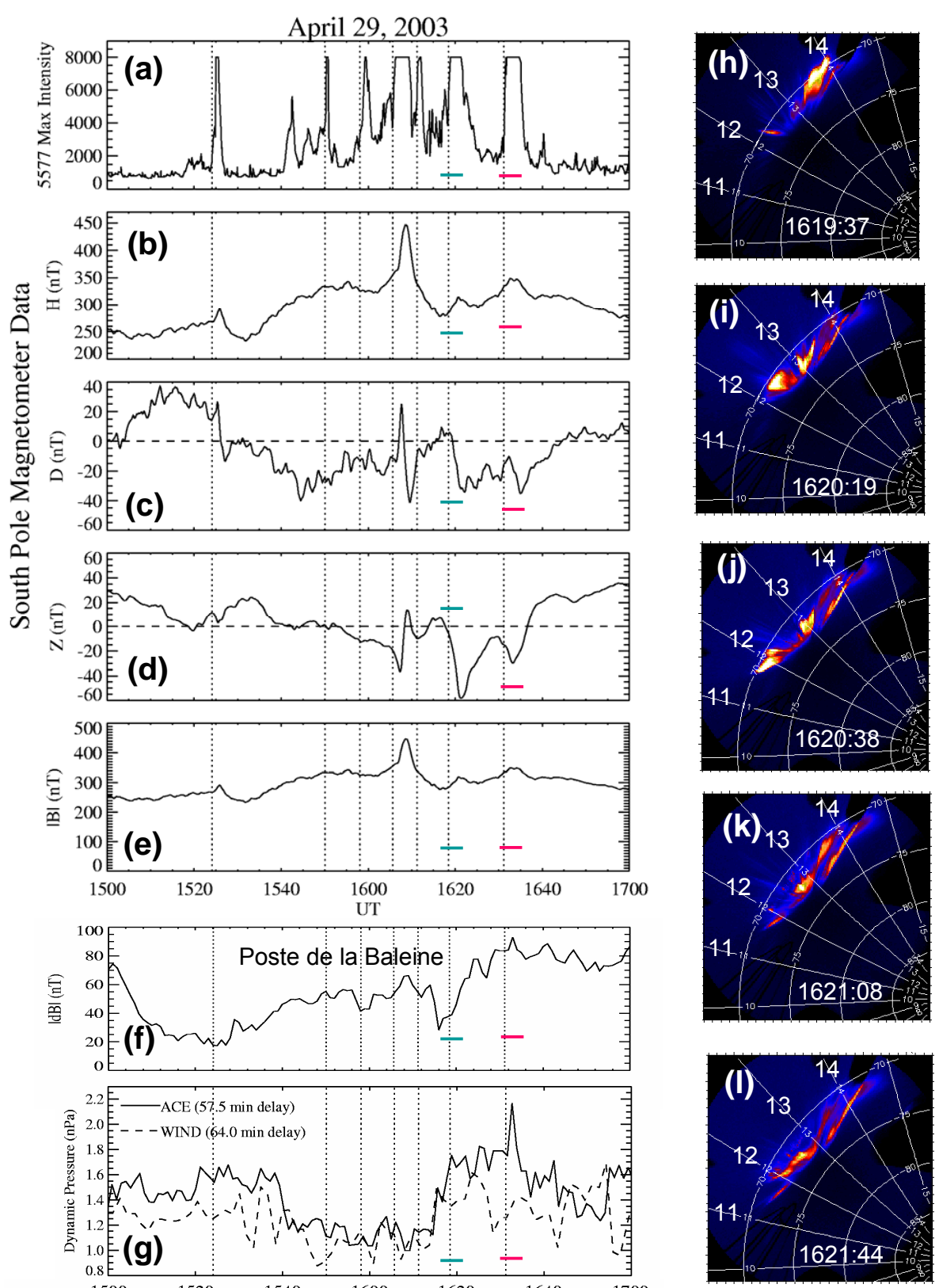

Fig. 14. Ground-based magnetometer measurements from South Pole Station and Poste de la Baleine, Quebec. Plotted are differences from values on a quiet reference day. (a) The maximum intensity of the $557.7 \mathrm{~nm}$ all-sky images from Fig. 6. (g) The dynamic pressure measured by ACE (solid line) and Wind (dashed line) properly lagged. (h-l) Selected all sky images from the interval marked by the green bars showing the response to a step function in dynamic pressure.

wind to show that the tilts of IEF phase planes can dramatically change in a few tens of minutes. Figure 3 illustrates how the tilt of phase plane can change with variations in IMF $B_{X}$ and consequently cause compressions/rarefactions in $B$ away from the hinge point. The very small negative variation in $|\mathbf{B}|$ in the lagged ACE data at 15:41 UT (Fig. 2) implies that ACE was near the point about which the phase plane was rotating. Propagating the measurements at ACE
(233, 40, $-21 R_{E}$ ) to $X=12 R_{E}$ using the solar wind velocity $(-540,-40,40 \mathrm{~km} / \mathrm{s}$ GSE) changes the applicable $Y$ and $Z$ coordinates to 23 and $-5 R_{E}$, respectively. The variations in $B_{X}$ observed at ACE were not seen at Wind about $40 R_{E}$ above ACE in the $Z$ direction. This means that the scale size of the variation was less than $40 R_{E}$, and the variations seen at Wind do not impact the magnetosphere (their $Z$ coordinate at the time it reached the proximity of the bow shock 
would have been more than $60 R_{E}$ ). ACE remains the best description of the IMF impacting the magnetopause, and the Cartoon of Fig. 3 is applicable, assuming the scale size is greater than the $20 R_{E}$ offset in $Y$.

Rapid increases in IMF $B_{X}$ and field magnitude near Cluster 4 at $\sim 15: 40$ UT and later at Cluster 1 indicate the enhancement is propagating toward the magnetopause (orange circle in Fig. 9h). The $|\mathbf{B}|$ enhancement occurred over twominutes, about twice as long as the rotation observed at ACE. The longer time for passing Cluster is an artifact of the different plasma speeds in the magnetosheath and solar wind as well as IMF draping over the magnetopause. During the IMF $B_{X}$ increase near 15:25 UT both Cluster 1 and Cluster 4 were in the magnetopause current layer where local variations in $|\mathbf{B}|$ obscured relatively small external changes. Note that the change in $B_{X}$ near 15:25 UT was not as rapid as at 15:40 UT. However, it was isolated from other interplanetary changes that affect merging rates. The increased $|\mathbf{B}|$ convecting into a separator region affects the local merging rate.

ISM simulations indicate responses that are widely distributed in local time and affect active merging sites beyond those conjugate to Cluster. They map last closed field lines between 09:00 and 10:00 MLT in the northern hemisphere to near magnetic noon in the southern ionosphere. For example, first open field line t (u) in Fig. 4 maps to 09:00 (12:00) MLT in the northern (southern) hemisphere. Strong $557.7 \mathrm{~nm}$ emissions observed at South Pole at 15:25 UT, coinciding with the $B_{X}$ change, started at noon (Fig. 121) and spread toward 11:30 MLT (Fig. 6b). The poleward movement of corresponding $630.0 \mathrm{~nm}$ emissions (Fig. 6c) was characteristic of a PMAF. A weak PMAF accompanied the 15:40 UT event. An electron tail signature detected by Cluster 4 at 15:25 UT (Fig. 12c) and $557.7 \mathrm{~nm}$ emissions near magnetic noon (Fig. 12l), suggest that the positive change in $B_{X}$ enhanced merging at multiple locations across a several prenoon hours in MLT.

\subsection{Clock-angle changes}

Maynard et al. (2004) and Sandholt et al. (2004) documented changes in the location and characteristics of auroral emissions as the IMF transitioned from $\theta \approx 135^{\circ}$ to $\sim 180^{\circ}$. As $\theta$ approached $180^{\circ} 557.7 \mathrm{~nm}$ emissions spread across noon but weakened. During winter-solstice observations at NyÅlesund PMAFs only occurred when $90^{\circ}<|\theta|<140^{\circ}$. Initial brightening occurred near the open-closed boundary in the prenoon (postnoon) sector for negative (positive) IMF $B_{Y}$ (Sandholt et al., 2004). This paper also documents emission responses to clock-angle changes, that are at times complicated by simultaneous changes in $P_{S W}$ and $B_{X}$.

Figure 11 shows a complex sequence of changes in $557.7 \mathrm{~nm}$ emissions attributable to changes in both $B_{X}$ and $\theta$. The event corresponds to intensity peaks 6 and 7 in Fig. 6 a. With $\theta \approx-135^{\circ}$ prenoon emissions were weak. The strongest emissions had ray structures with footprints between 13:00 and 14:00 MLT. We interpret the changed locations of intense $557.7 \mathrm{~nm}$ rays as signatures of recently activated merging sites rather than of filaments propagating away from the open-closed boundary. However, some features do not follow this interpretation strictly. Specifically, the initial brightening at 16:06 UT was near 13:30 MLT (Fig. 11c). Subsequently, bright emissions were observed poleward of the cusps equatorward boundary (cf. Fig. 6b) while $\theta$ applicable for the southern hemisphere was near $-120^{\circ}$. In succeeding images of Fig. 11 the brightest segment extended to $75^{\circ}$ MLat, several degrees poleward of the apparent equatorward boundary of the cusp (Fig. 11d). As the clock angle applicable to the northern hemisphere merging approached $180^{\circ}$, the brightest segment occurred in the prenoon sector and at lower latitudes (Fig. 11f), and appears to be a modulation of an event already in progress. The complexity of the event is magnified by: (1) different lag times applicable to the northern and the southern hemispheres, (2) IEF phaseplane tilting, (3) dipole tilt angle, and (4) increased southernhemisphere merging after a negative shift in IMF $B_{X}$.

Figures $11 \mathrm{e}$ and 1 show a region of reduced $557.7 \mathrm{~nm}$ emissions centered near 13:00 MLT, that is about $3^{\circ}$ wide in MLat and an hour in MLT. Emissions are also strong on the poleward side of a gap. This is not an artifact of the all-sky imager looking toward magnetic zenith. ISM simulations help to understand the source of these optical variations in the high-altitude cusp. Siscoe et al. (2005) identified the $\mathrm{YZ}$ plane containing the maximum cusp-diamagnetic depression for $0^{\circ}<\theta<180^{\circ}$ at $45^{\circ}$ intervals. Figure 15 shows shaded regions falling within the $-30 \mathrm{nT}$ contours and associated open-closed boundaries (adapted from Fig. 3 of Siscoe et al., 2005). Dashed and solid lines at the top of each panel respectively indicate the locations of magnetic noon and the centers of diamagnetic depressions. The cusp center moves from noon with $\theta=0^{\circ}$ toward dusk (dawn) for positive (negative) $B_{Y}$ to a maximum displacement for $B_{Z}=0$. It returns to a broad region centered about magnetic noon for pure $B_{Z}$ south. The $557.7 \mathrm{~nm}$ emissions shown in Fig. 11 displayed similar responses to IMF clock-angle changes, moving to later local times as $|\theta|$ decreased. Emissions appeared along the equatorward and dusk boundaries of the cusp as suggested in Figs. 15d and f. As $\theta$ approached $180^{\circ}$, the emissions appeared on both sides of noon in substantial conformity to Fig. 15h.

We suggest that $557.7 \mathrm{~nm}$ emissions gaps in Figs. 11e and 1 resemble in shape and location the cusp depression depicted by Siscoe et al. (2005) illustrated in Figs. 15f and g. Figure 16 emphasizes the emissions gap, as imaged at 16:07:53 UT $\left(\theta \approx-120^{\circ}\right), 16: 09: 05$ UT $\left(\theta \approx-135^{\circ}\right)$, and $16: 15: 37$ UT $\left(\theta \approx-180^{\circ}\right)$ with a factor of 2 higher gain. Green outlines of the gap provide shapes for comparison with the $-30 \mathrm{nT}$ contours in Figs. 15d, f, and to Fig. 11e, respectively. Note in Figs. 15 and 16 that the emissions gap migrated toward noon as $\theta$ increased in absolute value. For $180^{\circ}$ clock angles the emissions extended over more than $2 \mathrm{~h}$ 


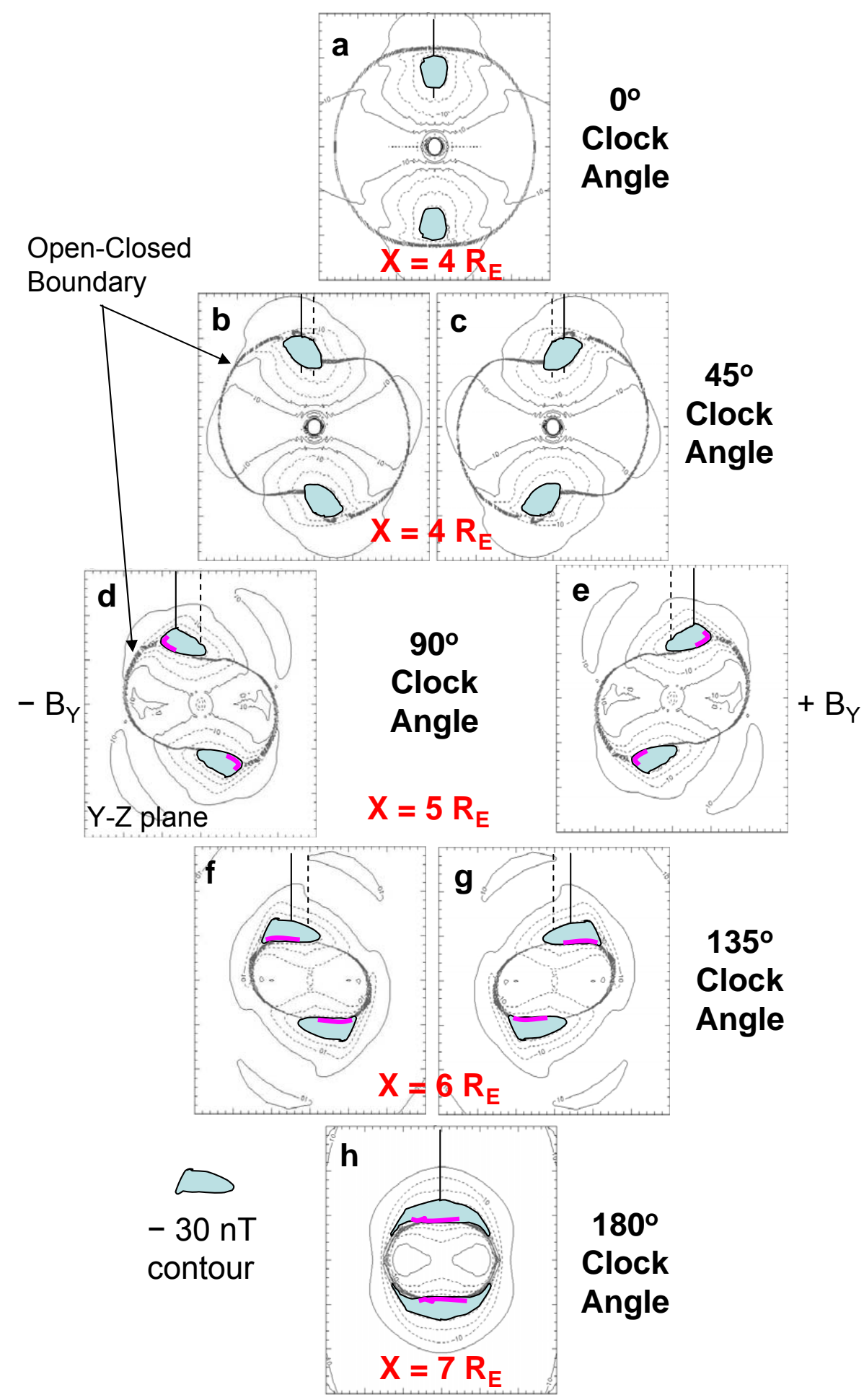

Fig. 15. An adaptation of Fig. 3 from Siscoe et al. (2005) showing contours of $\Delta B$ derived from ISM simulations using various clock angles but no dipole tilt. Plots show magnetic field differences from a dipole. The $Y Z$ plane is shown at the $X$ distance corresponding to the minimum cusp field. The $-30 \mathrm{nT}$ contour is shaded in to emphasize the cusps position. The red lines suggest locations of probable dayside merging might. The dashed and solid lines indicate local noon and the middle of the cusp depression, respectively. Note the cusp migration toward dusk (dawn) when positive (negative) IMF $B_{Y}$ dominates. 

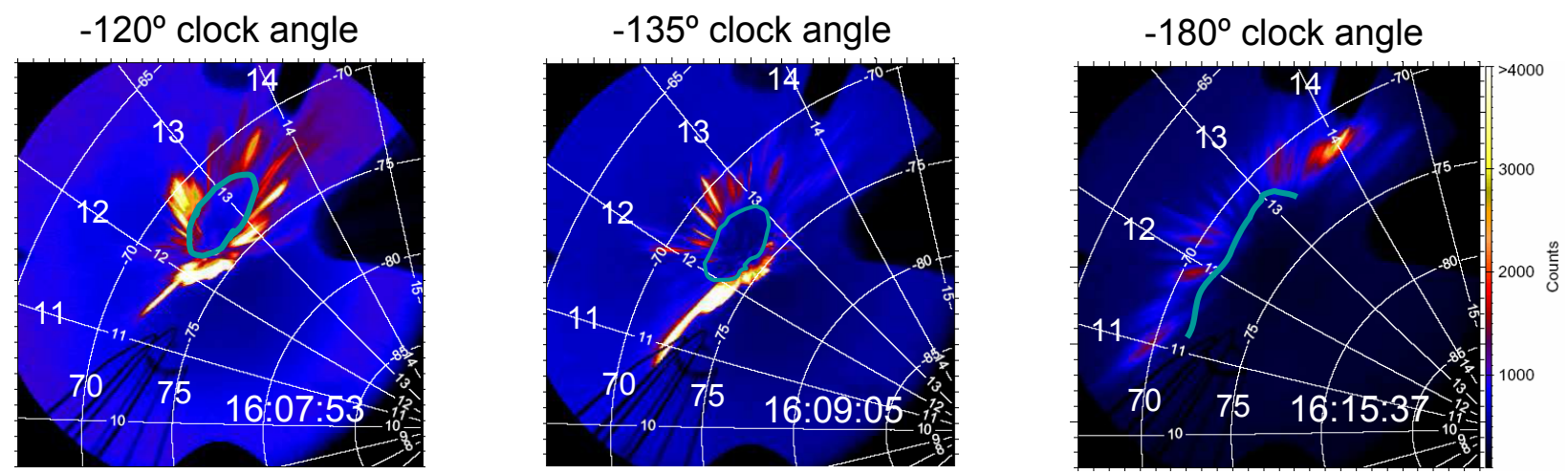

Fig. 16. Three $557.7 \mathrm{~nm}$ images for clock angles of $-120^{\circ},-135^{\circ}$, and $-180^{\circ}$ showing movement of the gap in emissions similar to the movement of the cusp depression in Fig. 15. The amplitude scale for the emissions is half that used in the other $557.7 \mathrm{~nm}$ images.

in MLT and were structured with longitude. No emissions from the poleward side of the cusp occur to define the gaps latitudinal extent.

$557.7 \mathrm{~nm}$ emissions shown in Figs. 16a and b were acquired at the time of the largest MIE recorded in Fig. 14. The eastward ionospheric current at magnetic noon corresponds to the most intense auroral emissions. Our conclusion that a localized current filament was moving poleward past South Pole Station is confirmed with the 557.7 and $630.0 \mathrm{~nm}$ keograms of Fig. 6. This event coincided with changes in IMF $B_{X}$ and the clock angle. The solar wind speed was high and the density low, similar to the majority of MIEs reported by Kataoka et al. (2003). Most of their events were associated with tangential discontinuities. However, most events also had small cone angles associated with hot flow anomalies (e.g., Schwartz et al., 2000). Also, 30\% of their events are associated with bursty merging, which is consistent with our interpretation of the reported events. In the present event $\theta$ was large and $B_{X}$ was approaching zero.

In agreement with our hypothesis that responsible electrons originate in merging events along the open-closed fieldline boundary, the $557.7 \mathrm{~nm}$ emissions primarily occur along the edge of the cusp depression. High-energy electrons drain from the newly opened flux tubes as they are dragged azimuthally and poleward through the cusp by the solar wind. Contrary to our hypothesis, Fig. 11d situates the most intense $557.7 \mathrm{~nm}$ emissions on the poleward edge of the cusp depression as described by Siscoe et al. (2005). However, at this time questions, regarding why the cusps poleward boundary should sometimes be marked by significant fluxes of $\mathrm{keV}$ electrons, cannot be answered with available measurements.

\section{Conclusions}

We have correlated $557.7 \mathrm{~nm}$ emissions observed in all-sky images from South Pole with solar wind and Cluster observations to investigate macro-scale properties solar wind/IMF driving of the dayside magnetosphere and ionosphere. En- ergetic electrons propagating along the separatrices from active merging sites are the sources of most emissions. As such they provide "television-like" pictures of the dayside merging. When the merging rate increases emissions intensify and poleward moving auroral forms (PMAFs) develop. We conclude or confirm the following from this study:

1. All-sky images from the South Pole Station confirm that merging occurs at multiple locations simultaneously and asynchronously (Maynard et al., 2004). At a particular location merging may be active for between tens of seconds to several minutes.

2. Most PMAFs reflect responses to changes in interplanetary properties, rather than local processes.

3. The changes in magnetopause position required by increases in dynamic pressure are mediated by merging and result in the generation of PMAFs. Small (15 to 20 percent) variations in dynamic pressure of the solar wind are sufficient to launch a PMAF.

4. Changes in IMF $B_{X}$ cause compressions and rarefactions of magnetic flux in the solar wind. The magnitude of increases at northern (southern) hemisphere merging sites for increases (decreases) in $B_{X}$, which enhances the merging rate and triggers a PMAF.

5. When $B_{X}$ is a significant component of the IMF, different lag times apply to northern and southern hemisphere merging sites (Maynard et al., 2001b).

6. Locations of dayside auroral emissions change with the IMF clock angle (Maynard et al. 2004). Increased clock angle can enhance merging rates and trigger PMAFs. However, at clock angles approaching $180^{\circ}$ emissions weaken, in agreement with an earlier finding that PMAFs generally occur at clock angles between $90^{\circ}$ and $140^{\circ}$ (Sandholt et al., 2004). 
7. In the presence of dipole tilt, merging sites are pushed toward the cusp opposite to the tilt for clock angles near $180^{\circ}$ (Maynard et al., 2003). From this study, the all-sky images (Figs. 11g and 16c) and the simulations (Fig. 10b, insert) show that merging occurs in multiple discrete locations, rather than continuously, across the dayside for southward IMF conditions.

Acknowledgements. We are grateful to C. W. Smith and D. J. McComas for use of the ACE magnetic field and plasma data, to R. Lepping and K. Ogilvie for use of the Wind magnetic field and plasma data, to H. Singer for use of the GOES data, and to M. Andre for use of the Cluster electric field data. The All-Sky Imager project at South Pole Station was conducted under an Agreement of Cooperation between Japanese National Institute of Polar Research and the United States National Science Foundation. The ISM was developed under sponsorship of the Defense Threat Reduction Agency, 45045 Aviation Drive, Dulles, VA 20166-7517. The magnetic field measurements at South Pole Station are supported by the Office of Polar Programs, NSF, under grant OPP-338105 to Siena College and the New Jersey Institute of Technology. The work at the University of New Hampshire and ATK Mission Research was supported by NASA through Cluster mission funding to the University of California at Berkeley (subcontracted). CJF is partially supported by NASA/SEC-GI grant NNG05GG25G. Work at Southwest Research Institute was also supported by NASA through Cluster mission funding.

Topical Editor I. A. Daglis thanks J. Vogt and another referee for their help in evaluating this paper.

\section{References}

Balogh, A., Carr, C. M., Acuña, M. H., Dunlop, M. W., Beek, T. J., Brown, P., Fornaon, K.-H., Georgescu, E., Glassmeier, K.H., Harris, J., Musmann, G., Oddy, T., and Schwingenschuh, K.: The Cluster magnetic field investigation: overview of in-flight performance and initial results, Ann. Geophys., 19, 1207-1217, 2001 , http://www.ann-geophys.net/19/1207/2001/.

Basinska, E. M., Burke, W. J., and Heinemann, M. A.: A user's guide to locating flux transfer events in low-altitude satellite measurements: An S3-2 case study, J. Geophys. Res., 94, 6681$6691,1989$.

Bosqued, J. M., Escoubet, C. P., Frey, H. U., Dunlop, M., Berchem, J., Marchaudon, A., Cerisier, J. C., Fazakerley, A., Budnik, E., Lavraud, B., Rème, H., Laakxso, H., and Balogh, A.: Multipoint observations of transient reconnection signatures in the cusp precipitation: A Cluster-IMAGE detailed case study, J. Geophys. Res., 110, A03219, doi:10.1029/2004JA010621, 2005.

Cerisier, J. C., Masrchaudon, A., Bosqued, J. M., McWilliams, K., Frey, H. U., Bouhram, M., Laakso, H., Dunlop, M., Forster, M., and Fazakerley, A.: Ionospheric signatures of plasma injections in the cusp triggered by solar wind pressure pulses, J. Geophys. Res., 110, A08204, doi:10.1029/2004JA010962, 2005.

Coleman, I. J., Chisham, G., Pinnock, M., and Freeman, M. P.: An ionospheric convection signature of antiparallel reconnection, J. Geophys. Res., 106, 28 995-29 007, 2001.

Cowley, S. W. H. and Lockwood, M.: Excitation and decay of solar wind driven flows in the magnetosphere-ionosphere system,
Ann. Geophys., 10, 103-115, 1992,

http://www.ann-geophys.net/10/103/1992/.

Crooker, N. U.: Dayside merging and cusp geometry, J. Geophys. Res., 84, 951-959, 1979.

Denig, W. F., Burke, W. J., Maynard, N. C., Rich, F. J., Jacobsen, B., Sandholt, P. E., Egeland, A., Leontjev, S., and Vorobjev, V. G.: Ionospheric signatures of dayside magnetopause transients: A case study using satellite and ground measurements, J. Geophys. Res., 98, 5969-5980, 1993.

Fasel, G. J.: Dayside poleward moving auroral forms: A statistical study, J. Geophys. Res., 11 891-11 905, 1995.

Friis-Christensen, E., McHenry, M. A., Clauer, C. R., and Vennerstrom, S.: Ionospheric traveling convection vortices observed near the polar cleft: A triggered response to sudden changes in the solar wind, Geophys. Res. Lett., 15, 253-256, 1988.

Gonzalez, W. D. and Mozer, F. S.: A quantitative model for the potential resulting from reconnection with arbitrary interplanetary magnetic field, J. Geophys. Res., 79, 4186-4194, 1974.

Gosling, J. T., Thomsen, M. F., Bame, S. J., Onsager, T. G., and Russell, C. T.: The electron edge of the low latitude boundary layer during accelerated flow events, Geophys. Res. Lett., 17, 1833-1836, 1990.

Gustafsson, G., Boström, R., Holback, B., Holmgren, G., Lundgren, A., Stasiewicz, K., Åhlén, Mozer, F. S. W., Pankow, D., Harvey, P., Berg, P., Ulrich, R., Pedersen, A., Schmidt, R., Butler, A., Fransen, A. W. C., Klinge, D., Thomsen, M., Fälthammar, C.-G., Lindqvist, P.-A., Christenson, S., Holtet, J., Lybekk, B., Sten, T. A., Tanskanen, P., Lappalainen, K., and Wygant, J.: The electric field and wave experiment for the Cluster mission, Space Sci. Rev., 79, 137-156, 1997.

Hain, K.: The partial donor cell method, J. Comp. Physics, 73, 131$147,1987$.

Johnstone, A. D., Alsop, C., Burge, S., Carter, P. J., Coates, A. J., Coker, A. J., Fazakerley, A. N., Grande, M., Gowen, R. A., Gurgiolo, C., Hancock, B. K., Narheim, B., Preece, A., Sheather, P. H., Winningham, J. D., and Woodliffe, R. D.: PEACE: A plasma electron and current experiment, Space Sci. Rev., 79, 351-398, 1997.

Katoaka, R., Fukunishi, H., Lanzerotti, L. J., Maclennan, C. G., Frey, H. U., Mende, S. B., Doolittle, J. H., Rosenberg, T. J., and Weatherwax, A. T.: Magnetic impulse events: A detailed case study of extended ground and space observations, J. Geophys. Res., 106, 25 873-25 889, 2001.

Kataoka, R., Fukunishi, H., and Lanzerotti, L. J.: Statistical identification of solar wind origins of magnetic impulse events, J. Geophys. Res., 108(A12), 1436, doi:10.1029/2003JA010202, 2003.

Kivelson, M. G. and Southwood, D. J.: Ionospheric traveling vortex generation by solar wind buffeting of the magnetosphere, J. Geophys. Res., 96, 1661-1667, 1991.

Konik, R. M., Lanzerotti, L. J., Wolfe, A., Maclennan, C. G., and Venkatesan, D.: Cusp latitude magnetic impulse events, 2. Interplanetary magnetic field and solar wind conditions, J. Geophys. Res., 99, 14 831-14 853, 1994.

Korotova, G. I., Sibeck, D. G., Moretto, T., and Reeves, G. D.: Tracking transient events through geosynchronous orbit, J. Geophys. Res., 104, 10 265-10 273, 1999.

Korotova, G. I., Sibeck, D. G., Singer, H. J., Rosenberg, T. J., and Engebretson, M. J.: Interplanetary magnetic field control of dayside transient event occurrence and motion in the ionosphere and 
magnetosphere, Ann. Geophys., 22, 4197-4202, 2004,

http://www.ann-geophys.net/22/4197/2004/.

Lanzerotti, L. J., Lee, L. C., Maclennan, C. G., Wolfe, A., and Medford, L. V.: Possible evidence of flux transfer events in the polar ionosphere, Geophys. Res. Lett., 11, 1089-1092, 1986.

Lepping, R. P., Acuña, M. H., Burlaga, L. F., Farrell, W. M., Slavin, J. A., Schatten, K. H., Mariani. F., Ness, N. F., Meubauer, F. M., Whang, Y. C., Byrnes, J. B., Kennon, R. S., Panetta, P. V., Scheifele, J., and Morley, E. M.: The Wind magnetic field investigation, Space Sci. Rev., 71, 207-229, 1995.

Lockwood, M.: Relationship of dayside auroral precipitations to the open-closed separatrix and patterns of convective flow, J. Geophys. Res., 102, 17 475-17 487, 1997.

Maynard, N. C.: Svalbard: A window for understanding tempo$\mathrm{ral} / \mathrm{sp}$ atial aspects of solar wind coupling to the magnetosphere and ionosphere, in: Egeland Symposium on Auroral and Atmospheric Research, edited by: Moen, J. and Holtet, J. A., p. 75-89, Department of Physics, University of Oslo, ISBN 82-91853-096, 2003.

Maynard, N. C.: Coupling the solar-wind/IMF to the ionosphere through the high latitude cusps, Surveys in Geophysics, 26, 255280, doi:10.1007/s10712-005-1882-4, 2005.

Maynard, N. C., Burke, W. J., Pfaff, R. F., Weber, E. J., Ober, D. M., Weimer, D. R., Moen, J., Milan, S., Sandholt, P. E., Egeland, A., Søraas. F., Lepping, R., Bounds, S., Acuña, M. H., Freudenreich, H., Gentile, L. C., Hardy, D. A., Holtet, J. A., Lester, M., Machuzak, J. S., Clemmons, J. H., Ning, P., Stadsnes, J., and van Eyken, T.: Driving dayside convection with northward IMF: Observations of a sounding rocket from Svalbard, J. Geophys. Res., 105, 5245-5263, 2000.

Maynard, N. C., Savin, S., Erickson, G. M., Kawano, H., Němeček, Z., Peterson, W. K., Šafránoková, J., Sandahl, I., Scudder, J. D., Siscoe, G. L., Sonnerup, B. U. Ö., Weimer, D. R., White, W. W., and Wilson, G. R.: Observation of the magnetospheric "sash" and its implications relative to solar-wind/magnetosphere coupling: A multisatellite event analysis, J. Geophys. Res., 106, 6097-6122, 2001a.

Maynard, N. C., Burke, W. J., Sandholt, P. E., Moen, J., Ober, D. M., Lester, M., Weimer, D. R., and Egeland, A.: Observations of simultaneous effects of merging in both hemispheres, J. Geophys. Res., 106, 24 551-24 577, $2001 \mathrm{~b}$.

Maynard, N. C., Ober, D. M., Burke, W. J., Scudder, J. D., Lester, M., Dunlop, M., Wild, J. A., Grocott, A., Farrugia, C. J., Lund, E. J., Russell, C. T., Weimer, D. R., Siebert, K. D., Balogh, A., Andre, M., and Rème, H.: Polar, Cluster and SuperDARN evidence for high latitude merging during southward IMF: temporal/spatial evolution, Ann. Geophys., 21, 2233-2258, 2003, http://www.ann-geophys.net/21/2233/2003/.

Maynard, N. C., Moen, J., Burke, W. J., Lester, M., Ober, D. M., Scudder, J. D., Siebert, K. D., Weimer, D. R., Russell, C. T., and Balogh, A.: Temporal-spatial structure of magnetic merging at the magnetopause inferred from 557.7-nm all-sky images, Ann. Geophys., 22, 2917-2942, 2004,

http://www.ann-geophys.net/22/2917/2004/.

Maynard, N. C., Burke, W. J., Scudder, J. D., Ober, D. M., Weimer, D. R., Siebert, K. D., Russell, C. T., Lester, M., Mozer, F. S., and Sato, N.: Electron signatures of active merging sites on the magnetopause, J. Geophys. Res., 110, A10207, doi:10.1029/2004JA010639, 2005.
McComas, D. J., Bame, S. J., Barber, P., Fieldman, W. C., Phillips, J. L., and Riley, P.: Solar wind electron, proton, and alpha monitor (SWEPAM) on the Advanced Composition Explorer, Space Sci. Rev., 86, 563-612, 1998.

Moore, T. E., Fok, M.-C., and Chandler, M. O.: The dayside reconnection X-line, J. Geophys. Res., 107(A10), 1332, doi:10.1029/2002JA009381, 2002.

Newell, P. T. and Meng, C. I.: Ionospheric projection of magnetospheric regions under low and high solar wind pressure conditions, J. Geophys. Res., 99, 273-286, 1994.

Ober, D. M., Maynard, N. C., Burke, W. J., Moen, J., Egeland, A., Sandholt, P. E., Farrugia, C. J., Weber, E. J., and Scudder, J. D.: Mapping prenoon auroral structures to the magnetosphere, J. Geophys. Res., 105, 27 519-27 530, 2000.

Ogilvie, K. W., Fitzenreiter, R. J., and Scudder, J. D.: Observations of electron beams in the low latitude boundary layer, J. Geophys. Res., 89, 10 723-10 732, 1984.

Ogilvie, K. W., Chornay, D. J., Fitzenreiter, R. J., Hunsaker, F., Keller, J., Lobell, J., Miller, G., Scudder, J. D., Sittler Jr., E. C., Torbert, R. B., Bodet, D., Needell, G., Lazarus, A. J., Steinberg, J. T., Tappan, J. H., Mavretic, A., and Gergin, E.: SWE: A comprehensive plasma instrument for the wind spacecraft: Space Sci. Rev., 71, 55-77, 1995.

Rees, M. H.: Auroral ionization and excitation by incident energetic electrons, Planet. Space Sci., 11, 1209-1218, 1963.

Rème, H., Aoustin, C., Bosqued, J. M., Dandouras, I., et al.: First multispacecraft ion measurements in and near the Earth's magnetosphere with the identical Cluster Ion Spectrometry (CIS) experiment, Ann. Geophys., 19, 1303-1354, 2001, http://www.ann-geophys.net/19/1303/2001/.

Roger, A. S., Chisham, G., Coleman, I. J., Freeman, M. P., and Pinnock, M.: Anti-parallel reconnection at the dayside magnetopause: Ionospheric signatures and implications for the low latitude boundary layer, in: Earths Low-Latitude Boundary Layer, Geophysical Monograph 133, edited by: Newell, P. T. and Onsager, T., pp. 311-318, American Geophysical Union, 2003.

Sandholt, P. E. and Farrugia, C. J.: Monitoring magnetosheathmagnetosphere interconnection topology from the aurora, Ann. Geophys., 20, 629-637, 2002,

http://www.ann-geophys.net/20/629/2002/.

Sandholt, P. E., Deehr, C. S., Egeland, A., Lybekk, B., Viereck, R., and Romick, G. J.: Signatures in the dayside aurora of plasma transfer from the magnetosheath, J. Geophys. Res., 91, $10063-$ $10079,1986$.

Sandholt, P. E., Farrugia, C. J., Burlaga, L. F., Holtet, J. A., Moen, J., Lybekk, B., Jacobsen, B., Opsvik, D., Egeland, A., Lepping, R., Lazarus, A. J., Hansen, T., Brekke, A., and Friis-Christensen, E.: Cusp/cleft auroral activity in relation to solar wind dynamic pressure, IMF $B_{Z}$ and $B_{Y}$, in: Physical Signatures of Magnetospheric Boundary Layer Processes, edited by: Holtet, J. A. and Egeland, A., pp. 173-188, Kluwer Academic Pub., Dordrecht, 1994.

Sandholt, P. E., Farrugia, C. J., Moen, J., Noraberg, Ø., Lybekk, B., Sten, T., and Hansen, T.: A classification of dayside auroral forms and activities as a function of interplanetary magnetic field orientation, J. Geophys. Res., 103, 23 325-23 345, 1998.

Sandholt, P. E., Denig, W. F., Farrugia, C. J., Lybekk, B., and Trondsen, E.: Auroral structure at the cusp equatorward boundary: relationship with the electron edge of the low latitude 
boundary layer precipitation, J. Geophys. Res., 107(A9), 1235, doi:10.1029/2001JA00581, 2002.

Sandholt, P. E., Farrugia, C. J., and Denig, W. F.: Dayside aurora and the role of IMF $\left|B_{Y}\right| /\left|B_{Z}\right|$ : detailed morphology and response to magnetopause reconnection, Ann. Geophys. 22, 613628, 2004.

Sanny, J., Sibeck, D. G., Venturinni, C. C., and Russell, C. T.: A statistical study of transient events in the outer dayside magnetosphere, J. Geophys. Res., 101, 4939-4952, 1996.

Schwartz, S. J., Paschmann, G., Sckopke, N., Bauer, T. M., Dunlop, M., Fazakerley, A. N., and Thomsen, M. F.: Conditions for the formation of hot flow anomalies at Earths bow shock, J. Geophys. Res., 105, 12 639-12 650, 2000.

Scudder, J. D., Mozer, F. S., Maynard, N. C., and Russell, C. T.: Fingerprints of collisionless reconnection at the separator: Ambipolar-Hall signatures, J. Geophys. Res., 107(A10), doi:10.1029/2001JA000126, 2002.

Sibeck, D. G.: A model for the transient magnetospheric response to sudden solar wind dynamic pressure variations, J. Geophys. Res., 95, 3755-3771, 1990.

Sibeck, D. G., Trivedi, N. B., Zesta, E., Decker, R. B., Singer, H. J., Szabo, A., Tachihara, H., and Watermann, J.: Pressure pulse interaction with the magnetosphere and ionosphere, J. Geophys. Res. 108(A2), 1095, doi:10.1029/2002JA009675, 2003.

Siscoe, G., Crooker, N., Siebert, K., Maynard, N., Weimer, D., and White, W.: Cusp geometry in MHD simulation, Surveys in Geophysics, 26, 387-407, doi:10.1007/s10712-005-1902-4, 2005.

Smith, C. W., Acuña, M. H., Burlaga, L. F., L'Heureux, J., Ness, N. F., and Scheifele, J.: The ACE Magnetic Field Experiment, Space Sci. Rev., 86, 613-632, 1998.
Sonnerup, B. U. Ö.: Magnetopause reconnection rate, J. Geophys. Res. 79, 1546-1549, 1974.

Sonnerup, B. U. Ö., Paschmann, G., Papamastorakis, I., Sckopke, N., Haerendel, G., Bame, S. J., Asbridge, J. R., Gosling, J. T., and Russell, C. T.: Evidence for magnetic reconnection at the earth's magnetopause, J. Geophys. Res., 86, 10 049-10 067, 1981.

Southward, D. J. and Kivelson, M. G.: The magnetohydrodynamic response to the magnetospheric cavity to changes in solar wind dynamic pressure, J. Geophys. Res., 95, 2301-2309, 1990.

Vaisberg, O. L., Smirnov, V. N., Avanov, L. A., Waite Jr., J. H., Burch, J. L., Gallagher, D. L., and Borodkova, N. L.: Different types of low-latitude boundary layer observed by Interball-Tail probe, J. Geophys. Res., 106, 13 067-13 090, 2001.

Weimer, D. R., Ober, D., Maynard, N. C., Burke, W. J., Collier, M. R., McComas, D. J., Ness, N. F., and Smith, C. W.: Variable time delays in the propagation of the interplanetary magnetic field, J. Geophys. Res., 107(A8), doi:10.1029/2001JA009102, 2002.

White, W. W., Siscoe, G. L., Erickson, G. M., Kaymaz, Z., Maynard, N. C., Siebert, K. D., Sonnerup, B. U. Ö., and Weimer, D. R.: The magnetospheric sash and cross-tail S, Geophys. Res. Lett., 25, 1605-1608, 1998.

White, W. W., Schoendorf, J. A., Siebert, K. D., Maynard, N. C., Weimer, D. R., Wilson, G. L., Sonnerup, B. U. Ö., Siscoe, G. L., and Erickson, G. M.: MHD simulation of magnetospheric transport at the mesoscale, in: Space Weather, Geophysical Monograph Series Vol. 125, edited by: Song, P., Singer, H. J., and Siscoe, G. L., pp. 229-240, American Geophys. Union, 2001.

Zesta, E., Hughes, W. J., Engebretson, M. J., Hughes, T. J., Lazarus, A. J., and Paulerina, K. I.: The November 9, 1993, traveling convection vortex event: A case study, J. Geophys. Res., 104, 28 041-28 058, 1999. 